\title{
Exponential Decay of Truncated Correlations for the Ising Model in any Dimension for all but the Critical Temperature
}

\author{
Hugo Duminil-Copin ${ }^{1,2}$, Subhajit Goswami ${ }^{2}$, Aran Raoufi ${ }^{3}$ \\ 1 Université de Genève, Geneva, Switzerland \\ 2 Institut des Hautes Études Scientifiques, Bures-sur-Yvette, France. E-mail: goswami@ihes.fr \\ 3 ETH Zurich, Zurich, Switzerland
}

Received: 12 October 2018 / Accepted: 5 October 2019

Published online: 28 November 2019 - (C) The Author(s) 2019

\begin{abstract}
The truncated two-point function of the ferromagnetic Ising model on $\mathbb{Z}^{d}$ $(d \geq 3)$ in its pure phases is proven to decay exponentially fast throughout the ordered regime $\left(\beta>\beta_{c}\right.$ and $\left.h=0\right)$. Together with the previously known results, this implies that the exponential clustering property holds throughout the model's phase diagram except for the critical point: $(\beta, h)=\left(\beta_{c}, 0\right)$.
\end{abstract}

\section{Introduction}

1.1. Exponential decay of truncated correlations of the Ising model. In addition to its original presentation as a model for the phase transition in ferromagnets, the Ising model has attracted attention from a variety of perspectives. These range from studies of phase transitions exhibited by the equilibrium states to the study of cutoff phenomena and transitions in stochastic processes given for instance by Glauber dynamics and Metropolis algorithms [LS13]. Also, universality of critical phenomena in the Ising model justifies the fact that the theory of the Ising model provides information also about many other systems.

As is well known, sufficiently far from phase transitions, systems of statistical physics exhibit exponential relaxation of truncated correlations [DS87], in both the equilibrium and the dynamical sense. It is more challenging to narrow the range of exceptions to a set of points, or lines, in the model's phase space. The main result in this article completes that task for the $d$-dimensional nearest-neighbor ferromagnetic Ising model. The results extend to finite-range Ising models, but we choose to focus on the nearest-neighbor case for simplicity.

To set the notation, let us recall the definition of the model on a graph $G$ with vertexset $V$ and edge-set $E$. Associated with the graph's vertex-set is a collection of binary variables $\sigma=\left(\sigma_{x}: x \in V\right)$, with $\sigma_{x} \in\{-1,1\}$. The system's Hamiltonian is given by 
the function

$$
H_{G, h}(\sigma):=-\sum_{x \in V} h \sigma_{x}-\sum_{\{x, y\} \subset V} J_{x, y} \sigma_{x} \sigma_{y},
$$

with the magnetic field $h$ and coupling constants $J_{x, y}$. In the case on which we focus here, $G$ equals $\mathbb{Z}^{d}$ (the graph is the regular $d$-dimensional lattice) and

$$
J_{x, y}= \begin{cases}1 & \text { if }\{x, y\} \in E \\ 0 & \text { otherwise }\end{cases}
$$

which corresponds to nearest-neighbor ferromagnetic interactions.

On finite graphs, the Gibbs equilibrium states at inverse temperature $\beta \in(0, \infty)$ are given by probability measures on the space of configurations under which the expected value of a function $f:\{-1,1\}^{V} \rightarrow \mathbb{R}$ is

$$
\langle f\rangle_{G, \beta, h}=\frac{1}{Z(G, \beta, h)} \sum_{\sigma \in\{-1,1\}^{V}} f(\sigma) \exp \left[-\beta H_{G, h}(\sigma)\right],
$$

where the sum is normalized by the partition function $Z(G, \beta, h)$ so that $\langle 1\rangle_{G, \beta, h}=1$. Examples of Gibbs measures on $\mathbb{Z}^{d}$ can be constructed as weak limits of the finite volume Gibbs measures on finite subgraphs $G \subset \mathbb{Z}^{d}$ which locally converge to $\mathbb{Z}^{d}$. We denote the measure thus obtained by $\langle\cdot\rangle_{\beta, h}$. Also, one defines

$$
\langle\cdot\rangle_{\beta}^{+}=\lim _{h \searrow 0}\langle\cdot\rangle_{\beta, h}
$$

where the limit is meant in the "weak sense" (i.e. for the expectation values of local functions of the spins). Convergence can be deduced by monotonicity arguments based on correlation inequalities, by which one may also establish the existence of $\beta_{c}=$ $\beta_{c}\left(\mathbb{Z}^{d}\right) \in[0, \infty]$ such that

$$
\begin{array}{r}
0 \leq \beta<\beta_{c} \Rightarrow\left\langle\sigma_{x}\right\rangle_{\beta}^{+}=0, \quad \forall x \in \mathbb{Z}^{d}, \\
\beta>\beta_{c} \Rightarrow\left\langle\sigma_{x}\right\rangle_{\beta}^{+}>0, \quad \forall x \in \mathbb{Z}^{d} .
\end{array}
$$

For a given Gibbs measure $\langle\cdot\rangle$, in finite or infinite volume, the truncated two-point correlation function is defined as:

$$
\left\langle\sigma_{0} ; \sigma_{x}\right\rangle:=\left\langle\sigma_{0} \sigma_{x}\right\rangle-\left\langle\sigma_{0}\right\rangle\left\langle\sigma_{x}\right\rangle
$$

For $\beta>\beta_{c}$, there exists a spin-flip symmetric equilibrium state with long-range order, for which the truncated correlations do not decay to zero. However, the relevant question is the rate of decay of the pure state $\langle\cdot\rangle_{\beta}^{+}$and its symmetric image $\langle\cdot\rangle_{\beta}^{-}$. The main result of this article is the following.

Theorem 1.1. For the nearest-neighbor Ising model on $\mathbb{Z}^{d}$ in dimension $d \geq 3$, for any $\beta>\beta_{c}$ there exists $c=c(\beta, d)>0$ such that for every $x, y \in \mathbb{Z}^{d}$,

$$
0 \leq\left\langle\sigma_{x} ; \sigma_{y}\right\rangle_{\beta}^{+} \leq \exp [-c\|x-y\|]
$$


The previous result holds for any extremal translation invariant Gibbs state, since by [Bod05, Rao17], those are given by $\langle\cdot\rangle_{\beta}^{+}$and $\langle\cdot\rangle_{\beta}^{-}$. As mentioned above, the theorem would extend to finite-range interactions, but we prefer to focus on this context for simplicity of notations. Jointly with the previously known results, this completes the proof that for the nearest-neighbor Ising model in any dimension it is only at the critical point $(h, \beta)=$ $\left(0, \beta_{c}\right)$ that the pure state's truncated two-point function fails to decay exponentially fast. The aforementioned statement which Theorem 1.1 supplements include:

1. At any $h \neq 0$ the limiting state is analytic in $h$ and $\beta$, and it exhibits exponential decay of suitably truncated correlations. This was proven by Lebowitz and Penrose in [LP68] using an argument which drew on the model's Lee-Yang property [LY52], or using the random-current representation.

2. For $h=0$ and $\beta<\beta_{c}$ the exponential decay in arbitrary dimension was established in [ABF87] (see also [DT16] for an alternative proof).

3. In the converse direction: the vanishing of the spontaneous magnetization at $(h, \beta)=$ $\left(0, \beta_{c}\right)$ for the nearest neighbor model in any dimension [ADCS15] together with the lower bound

$$
\sum_{\|x\|_{\infty}=R}\left\langle\sigma_{0} \sigma_{x}\right\rangle_{\beta_{c}} \geq 1
$$

which was established by Simon [Sim80], imply that for any $d \geq 2$ at the critical point the truncated two-point function does not decay exponentially fast.

4. And, to mention a last result: the special case of $d=2$ is analyzable through Onsager's exact solution [MW73] or using the Kramers-Wannier duality (the decay of truncated correlations can be obtained via the decay of correlations in the hightemperature dual Ising model).

Let us add that the truncated two-point function offers a bound on the decay of more general correlations which follows easily from the following lemma (whose proof follows from the switching lemma; see the discussion in Sect. 2.2). For a set of vertices $A$, set $\sigma_{A}:=\prod_{x \in A} \sigma_{x}$.

Lemma 1.2. For every finite graph $G$, every $\beta>\beta_{c}$ and every two disjoint sets of vertices $A$ and $B$, we have that

$$
0 \leq\left\langle\sigma_{A} \sigma_{B}\right\rangle_{G, \beta}^{+}-\left\langle\sigma_{A}\right\rangle_{G, \beta}^{+}\left\langle\sigma_{B}\right\rangle_{G, \beta}^{+} \leq 2^{|A|+|B|-4} \sum_{\substack{a \in A \\ b \in B}}\left\langle\sigma_{a} ; \sigma_{b}\right\rangle_{G, \beta}^{+} .
$$

1.2. Mixing of the Fortuin-Kasteleyn representation of the Ising model. Theorem 1.1 will be derived by studying a related model, called the Fortuin-Kasteleyn (FK) percolation, or random-cluster model. FK percolation is one of the most classical generalizations of Bernoulli percolation and electrical networks. This model was introduced by Fortuin and Kasteleyn in [FK72] and since then has been the object of intense studies, both physically and mathematically.

A percolation configuration on a graph $G=(V, E)$ is an element $\omega=\left(\omega_{e}: e \in E\right)$ in $\{0,1\}^{E}$. An edge $e$ is said to be open (in $\omega$ ) if $\omega_{e}=1$, otherwise it is closed. A configuration $\omega$ can be seen as a subgraph of $G$ with vertex-set $V$ and edge-set $\left\{e \in E: \omega_{e}=1\right\}$. A cluster is a connected component of the graph $\omega$. Below, we will write $x \leftrightarrow y$ if $x$ and $y$ are in the same cluster, and $x \leftrightarrow \infty$ if $x$ is in an infinite cluster. 
Fix $p \in[0,1]$ and $q \geq 1$. Let $G$ be a finite subgraph of $\mathbb{Z}^{d}$ and $\xi$ a configuration on $\mathbb{Z}^{d}$. Let $\phi_{G, p, q}^{\xi}$ be the measure on percolation configurations $\omega$ on $G$ defined by

$$
\phi_{G, p, q}^{\xi}(\omega)=\frac{1}{Z^{\xi}(G, p, q)}\left(\frac{p}{1-p}\right)^{|\omega|} q^{k_{\xi}(\omega)},
$$

where $|\omega|:=\sum_{e \in E} \omega_{e}$ and $k_{\xi}(\omega)$ is the number of clusters intersecting $G$ of the percolation configuration $\bar{\omega}$ on $\mathbb{Z}^{d}$ defined by $\bar{\omega}_{e}=\omega_{e}$ if $e \in E$, and $\xi_{e}$ if $e \notin E$, and $Z^{\xi}(G, p, q)$ is a normalizing constant making the total mass of the measure equal to 1 . We refer to $\xi$ as the boundary condition of $\phi_{G, p, q}^{\xi}$. In the particular case when $\xi \equiv 1$ (or 0 ) we denote the corresponding measure by $\phi_{G, p, q}^{1}$ (respectively $\phi_{G, p, q}^{0}$ ) and call the corresponding boundary condition as wired (respectively free).

The FK-percolation model with cluster-weight $q=2$ is related to the Ising model via the Edwards-Sokal coupling (see next section) and is therefore referred to in this article as the FK-Ising model. When $q=2$, it was proved in [Bod06, Rao17] that for every $p \in[0,1]$, there exists a unique infinite-volume measure $\phi_{p, 2}$ which is the weak limit of measures $\phi_{G, p, 2}^{\xi}$ as $G$ exhausts $\mathbb{Z}^{d}$. Furthermore, there exists a constant $p_{c}=p_{c}(d)$ such that $\phi_{p, 2}[0 \leftrightarrow \infty]$ is equal to 0 for every $p<p_{c}$, and is strictly positive for every $p>p_{c}$. We refer to [Gri06] for a justification that this limit exists.

Below and in the rest of this paper, we focus on the case $q=2$ and drop it from the notation. We denote $x+[-n, n]^{d} \cap \mathbb{Z}^{d}$ by $\Lambda_{n}(x)$ and the set of edges between two vertices of $\Lambda_{n}(x)$ by $E_{n}(x)$. In the particular case when $x=0$, we write $\Lambda_{n}$ and $E_{n}$ respectively. The boundary of $\Lambda_{n}(x)$, denoted as $\partial \Lambda_{n}(x)$, is defined as the set of all vertices in $\Lambda_{n}(x)$ which have a neighbor in $\mathbb{Z}^{d} \backslash \Lambda_{n}(x)$. Theorem 1.1 is a consequence of the following exponential mixing property.

Theorem 1.3 (Exponential mixing). For every $d \geq 3$ and $p>p_{c}$, there exists a constant $c>0$ such that for every $n \geq 1$,

$$
\left|\phi_{p}[A \cap B]-\phi_{p}[A] \phi_{p}[B]\right| \leq \exp (-c n),
$$

where $A$ and $B$ are any two events depending on edges in $E_{n}$ and outside $E_{2 n}$ respectively.

Before discussing the proof of this theorem, let us explain how it implies Theorem 1.1. Proof of Theorem 1.1. Fix $\beta>\beta_{c}$ and set $p:=1-e^{-2 \beta}>p_{c}$. The Edwards-Sokal coupling [see (2.6) in the next section] gives that for every $x \in \mathbb{Z}^{d}$,

$$
\left\langle\sigma_{0} ; \sigma_{x}\right\rangle_{\beta}^{+}=\left\langle\sigma_{0} \sigma_{x}\right\rangle_{\beta}^{+}-\left\langle\sigma_{0}\right\rangle_{\beta}^{+}\left\langle\sigma_{x}\right\rangle_{\beta}^{+}=\phi_{p}[0 \leftrightarrow x]-\phi_{p}[0 \leftrightarrow \infty] \phi_{p}[x \leftrightarrow \infty] .
$$

Assuming that $x$ is at a graph distance of at least $4 n$ of the origin, this implies that

$$
\begin{aligned}
\left\langle\sigma_{0} ; \sigma_{x}\right\rangle_{\beta}^{+} & \leq \phi_{p}\left[0 \leftrightarrow \partial \Lambda_{n}, x \leftrightarrow \partial \Lambda_{n}(x)\right]-\phi_{p}\left[0 \leftrightarrow \partial \Lambda_{n}\right] \phi_{p}\left[x \leftrightarrow \partial \Lambda_{n}(x)\right]+2 e^{-c^{\prime} n} \\
& \leq \mathrm{e}^{-c n}+2 \mathrm{e}^{-c^{\prime} n}
\end{aligned}
$$

where in the second line, we used Theorem 1.3, and in the first, the fact that for $p>p_{c}$, there exists $c^{\prime}>0$ such that for every $n \geq 1$,

$$
\phi_{p}\left[0 \leftrightarrow \partial \Lambda_{n}, 0 \leftrightarrow \infty\right] \leq \mathrm{e}^{-c^{\prime} n} .
$$

(This fact follows from Theorem (5.104) of [Gri06] when combined with the result of [Bod05].) 
Let us remark that the exponential decay (1.10) for the size of finite clusters in the supercritical FK-Ising model does not directly imply the exponential decay of truncated two-point functions for the Ising model, since the first term on the right of (1.9) involves correlations between the events that $0 \leftrightarrow \partial \Lambda_{n}$ and $x \leftrightarrow \partial \Lambda_{n}(x)$, and that these correlations could a priori be large.

Let us also remark that our method actually gives a better bound on the error term in Theorem 1.3 than $\exp (-c n)$. Namely, we obtain that

$$
\left|\phi_{p}[A \cap B]-\phi_{p}[A] \phi_{p}[B]\right| \leq \exp (-c n) \max _{\xi} \phi_{\Lambda_{n}, p}^{\xi}[A] \phi_{p}[B] .
$$

This is stronger than the weak mixing property for FK percolation measures which is obtained by replacing $\max _{\xi} \phi_{\Lambda_{n}, p}^{\xi}[A]$ with 1 but weaker than the ratio weak mixing property where we want to get rid of the maximum over boundary conditions. However our proof of Theorem 1.3 (see, e.g., (3.4)) also implies that $\phi_{p}$ has the so-called exponentially bounded controlling regions in the sense of [Ale98, p. 455]. Then the ratio weak mixing property of $\phi_{p}$ follows from (1.11) and Theorem 3.3. in [Ale98]. For potential application in future works we present it here as a corollary of Theorem 1.3.

Corollary 1.4 (Ratio weak mixing). For every $d \geq 3$ and $p>p_{c}$, there exists a constant $c>0$ such that for every $n \geq 1$,

$$
\left|\phi_{p}[A \cap B]-\phi_{p}[A] \phi_{p}[B]\right| \leq \exp (-c n) \phi_{p}[A] \phi_{p}[B],
$$

where $A$ and $B$ are any two events depending on edges in $E_{n}$ and outside $E_{2 n}$ respectively.

1.3. Idea of the proof. The core of the proof will be the derivation of the following proposition.

Proposition 1.5. There exists $c>0$ such that for every integer $N$ that is divisible by 4 ,

$$
\max _{e \in E_{N / 4}} \phi_{\Lambda_{N}, p}^{1}\left[\omega_{e}\right]-\phi_{\Lambda_{N}, p}^{0}\left[\omega_{e}\right] \leq \exp \left[-c(\log N)^{1+c}\right]
$$

The important feature of the upper bound above is that it beats any inverse polynomial. This proposition, together with a coarse-grained argument inspired by Pisztora, implies the exponential mixing. While the Ising model can be approached through a number of graphical representations (low and high temperature expansions, FK-Ising, random current etc.), which have been used separately in a variety of results, the argument presented here relies in a crucial way on the combination of two such representations: the random current and the FK-Ising. The random current representation is used to rewrite the difference between $\phi_{\Lambda_{N}, p}^{1}\left[\omega_{e}\right]$ and $\phi_{\Lambda_{N}, p}^{0}\left[\omega_{e}\right]$ in terms of the probabilities of non-intersection for currents in a duplicated system of currents. Then, FK-Ising is used to show that this duplicated system of currents is very well-connected, and that the probability of long paths of currents not being connected is quite small.

At different stages of the proof (already in the proof of Theorem 1.1 above), essential use is made of the very helpful result of Bodineau [Bod05] stating that for any $d \geq 3$ the critical parameter $p_{c}$ coincides with the so-called slab percolation. This result is combined with the result [Pis96] to implement a coarse-grain argument inspired by Pisztora renormalization. This is used to prove two facts: boxes are connected with excellent probability in the supercritical FK-Ising model, and Theorem 1.3 follows from Proposition 1.5. 
1.4. Open problems. Corollary 1.4 falls short of the ratio-strong mixing property related to the phenomenon of boundary phase transition for Ising models (see [MOS94]). Although this stronger property is absent for Ising models in dimensions larger than 2 at low temperature, it is expected to hold in the entire subcritical phase. More precisely, one would like to prove:

$$
\left|\phi_{p}[A \cap B]-\phi_{p}[A] \phi_{p}[B]\right| \leq \exp \left(-c d_{A, B}\right) \phi_{p}[A] \phi_{p}[B]
$$

where $d_{A, B}$ is the distance between the supports of the events $A$ and $B$.

Another important improvement would be to understand the case of the Potts models with $q \geq 3$ colors. While the $\beta<\beta_{c}$ was recently treated in every dimension in [DRT17], the study of the $\beta>\beta_{c}$ regime is still very limited. In [DT17], a partial result going in the direction of the equivalent of [Bod05] for Potts model was obtained. We refer to the paper for details on open questions and conjectures. Bodineau's result being the key to our argument (not to mention the heavy use of the random-current representation, which itself is not available for the Potts model), we believe that the exponential decay of correlations would be even harder to obtain than the open problems mentioned in [DT17].

Organization. The paper is organized as follows. In the next section, we recall some background. In Sect. 3, we present the coarse-graining arguments relying on Pisztora's technique. In Sect. 4, we prove Proposition 1.5, conditionally on two technical statements which are proved in Sect. 5.

\section{Background}

2.1. The FK-Ising model. We will use a few properties of the FK-Ising model that we recall now. For details and proofs, we direct the reader to [Gri06,Dum17].

Spatial Markov property. Let $H \subset G$ be two finite subgraphs of $\mathbb{Z}^{d}$ with respective edge-sets $E$ and $F$. A configuration $\omega$ on $G$ may be viewed as a configuration on $H$ by taking its restriction $\omega_{\mid E}$. The restriction of the configuration $\omega$ to edges of $F \backslash E$ induces boundary conditions on $G$. Namely, the spatial Markov property states that for any $p, q$ and any configuration $\xi$,

$$
\phi_{G, p}^{\xi}\left(\omega_{\mid E}=\cdot \mid \omega_{e}=\xi_{e}, \forall e \in F \backslash E\right)=\phi_{H, p}^{\xi}(\cdot) .
$$

The spatial Markov property implies the following finite-energy property: for every $\xi$,

$$
\frac{p}{2-p} \leq \phi_{\{e\}, p}^{\xi}\left[\omega_{e}\right] \leq p .
$$

Stochastic ordering for. $q \geq 1$. For any finite graph $G$, the set $\{0,1\}^{E}$ has a natural partial order. An event $A$ is increasing if for every $\omega \leq \omega^{\prime}, \omega \in A$ implies $\omega^{\prime} \in A$. The FK-Ising model satisfies the following properties. Fix $p \in[0,1]$ and $\xi \leq \xi^{\prime}$,

1. (FKG inequality) For every two increasing events $A$ and $B$,

$$
\phi_{G, p}^{\xi}[A \cap B] \geq \phi_{G, p}^{\xi}[A] \phi_{G, p}^{\xi}[B] .
$$


2. (Comparison between boundary conditions) For every increasing event $A$,

$$
\phi_{G, p}^{\xi^{\prime}}[A] \geq \phi_{G, p}^{\xi}[A]
$$

This last condition, together with (2.1), enables one to construct measures $\phi_{p}^{1}$ and $\phi_{p}^{0}$ in $\mathbb{Z}^{d}$ as weak limits of measures with free and wired boundary conditions in finite volume. It was proved in [Bod06] (see also [Rao17]) that $\phi_{p}^{1}=\phi_{p}^{0}$ for every $p \neq p_{c}$ (see [ADCS15] for the case $p=p_{c}$ ), and this is the reason why we refer to the infinite-volume measure as simply $\phi_{p}$.

Remark 2.1. We will often consider couplings between two FK-Ising measures. In this case, we will use the spatial Markov property and the FKG inequality applied to both configurations at the same time, two properties that we will call joint Markov property and joint FKG inequality. These properties will be justified by the standard spatial Markov property and the FKG inequality combined with the special constructions of these measures. Since the justification is classical, we will omit it in this article.

Edwards-Sokal coupling. We will use the Edwards-Sokal coupling both in finite and infinite volume. On a finite graph, the coupling goes as follows. Consider $\beta$ and $p$ related by $p=1-e^{-2 \beta}$. Consider a configuration $\omega$ sampled according to $\phi_{G, p}^{0}$ and assign to each cluster $\mathcal{C}$ of $\omega$ a spin $\sigma_{\mathcal{C}}$ in $\{-1,+1\}$ uniformly and independently for each cluster. Then, set $\sigma_{x}=\sigma_{\mathcal{C}}$ for every $x \in \mathcal{C}$. As a direct consequence of this coupling, one obtains that

$$
\left\langle\sigma_{A}\right\rangle_{G, \beta}=\phi_{G, p}^{0}\left[\mathcal{F}_{A}\right]
$$

where $\mathcal{F}_{A}$ is the event that every cluster of $\omega$ intersects an even number of times the set $A$. Note that when $A=\{x, y\}$, this translates into $\left\langle\sigma_{x} \sigma_{y}\right\rangle_{G, \beta}=\phi_{G, p}^{0}[x \leftrightarrow y]$.

We will also use the coupling in infinite volume. In this case, one can consider $\phi_{p}$ and assign a spin to each one of the finite clusters at random as explained previously, and a spin + to the infinite clusters (there is in fact at most one such cluster). One then obtains the measure $\langle\cdot\rangle_{\beta}^{+}$. Altogether, we deduce from this representation that

$$
\left\langle\sigma_{x}\right\rangle_{\beta}^{+}=\phi_{p}[x \leftrightarrow \infty] .
$$

We have in particular that $\beta_{c}=\frac{1}{2} \log \left(1-p_{c}\right)$.

Griffiths inequality. The monotonicity properties of the FK-Ising model imply the following two classical inequalities, which will be very useful: for every sets of vertices $A$ and $B$,

$$
\left\langle\sigma_{A} \sigma_{B}\right\rangle_{G, \beta}^{+} \geq\left\langle\sigma_{A}\right\rangle_{G, \beta}^{+}\left\langle\sigma_{B}\right\rangle_{G, \beta}^{+}
$$

and, if exceptionally we consider the model with arbitrary coupling constants $J$ (we refer to $J$ in the notation by writing $\langle\cdot\rangle_{G, J, \beta}$ for the measure), we have that for every coupling constants $J \geq J^{\prime} \geq 0$,

$$
\left\langle\sigma_{A}\right\rangle_{G, J, \beta} \geq\left\langle\sigma_{A}\right\rangle_{G, J^{\prime}, \beta}
$$


2.2. The random-current representation. We will also use the random-current representation in several places. A current configuration $\mathbf{n}$ on a graph $G$ with vertex-set $V$ and edge-set $E$ is an integer valued function on $E$, i.e. a function $\mathbf{n}: E \mapsto \mathbb{Z}_{+}$. A source of $\mathbf{n}=(\mathbf{n}(x, y):\{x, y\} \in E)$ is a vertex $x$ for which $\Delta_{x}(\mathbf{n}):=\sum_{y \in V: y \sim x} \mathbf{n}(x, y)$ is odd. The set of sources of $\mathbf{n}$ is denoted by $\partial \mathbf{n}$. The random current configuration's weight, at specified $\beta>0$, is given by

$$
w_{\beta}(\mathbf{n}):=\prod_{\{x, y\} \in E} \frac{\beta^{\mathbf{n}(x, y)}}{\mathbf{n}(x, y) !} .
$$

For every finite subgraph $G$ of $\mathbb{Z}^{d}$, we also construct a graph $G^{+}=\left(V^{+}, E^{+}\right)$with $V^{+}=V \cup\{\mathfrak{g}\}$, where $\mathfrak{g}$ is called the ghost vertex, and $E^{+}$is the union of $E$ together with as many edges $\{x, \mathfrak{g}\}$ as edges between $x$ and a vertex of $\mathbb{Z}^{d}$ outside of $G$. Note that there can be multiple edges between two given vertices in $G^{+}$, but that only vertices on the boundary (i.e. the vertices neighboring a vertex in $\mathbb{Z}^{d} \backslash V$ ) of $G$ can be connected to the ghost vertex.

Correlations of the Ising model can be expressed in terms of the random-current representation via the following formula: for every $A \subset V$,

$$
\left\langle\sigma_{A}\right\rangle_{G, \beta}:=\frac{\sum_{\mathbf{n} \in \mathbb{Z}_{+}^{E}: \partial \mathbf{n}=A} w_{\beta}(\mathbf{n})}{\sum_{\mathbf{n} \in \mathbb{Z}_{+}^{E}: \partial \mathbf{n}=\emptyset} w_{\beta}(\mathbf{n})} \text { and }\left\langle\sigma_{A}\right\rangle_{G, \beta}^{+}:=\frac{\sum_{\mathbf{n} \in \mathbb{Z}_{+}^{E^{+}}: \partial \mathbf{n} \cap V=A} w_{\beta}(\mathbf{n})}{\sum_{\mathbf{n} \in \mathbb{Z}_{+}^{E^{+}}: \partial \mathbf{n} \cap V=\emptyset} w_{\beta}(\mathbf{n})} .
$$

Note that the random-current representation of spin correlations for free boundary conditions involve currents on $G$, while those for plus boundary conditions involve currents on $G^{+}$.

The great utility of the random current representation results from a switching symmetry, whose roots lie in a combinatorial identity of [GHS70]. Using this symmetry, the Ising phase transition was presented in [Aiz82] as a phenomenon of percolation in a system of current loops. Resulting relations have been instrumental in shedding light on the critical behavior of the model in various dimensions [Aiz82, ABF87, AF86, ADCS15,ADTW18]. We do not wish to state the switching lemma here and refer to the corresponding literature. Rather, we present the applications we will need for our study.

To express various correlation functions (of finite systems) in terms of probabilities for systems of currents with prescribed sources, we introduce the probability measure $\mathbb{P}_{G}^{A}$ on currents $\mathbf{n} \in \mathbb{Z}_{+}^{E}$ with $\partial \mathbf{n}=A$ by the formula

$$
\mathbb{P}_{G}^{A}[\{\mathbf{n}\}]:=\frac{w_{\beta}(\mathbf{n})}{\sum_{\mathbf{m} \in \mathbb{Z}_{+}^{E}: \partial \mathbf{m}=A} w_{\beta}(\mathbf{m})} .
$$

Similarly, one defines $\mathbb{P}_{G^{+}}^{A}$ on currents $\mathbf{n} \in \mathbb{Z}_{+}^{E_{+}}$with $\partial \mathbf{n} \cap V=A$. Let $\mathbb{P}_{G^{+}, G}^{A, \varnothing}$ (resp. $\mathbb{P}_{G^{+}, G^{+}}^{A, \varnothing}$ ) denote the law of two independent currents with respective laws $\mathbb{P}_{G^{+}}^{A}$ and $\mathbb{P}_{G}^{\emptyset}$ (resp. $\mathbb{P}_{G^{+}}^{\emptyset}$ ). The key relation of interest, which, at the risk of repeating ourselves, is a consequence of the switching lemma, is the following:

$$
\mathbb{P}_{G^{+}, G}^{A, \emptyset}\left[x \underset{G}{\stackrel{\mathbf{n}_{1}+\mathbf{n}_{2}}{\rightleftarrows}} y\right]=\frac{\left\langle\sigma_{A} \sigma_{x} \sigma_{y}\right\rangle_{G}^{+}\left\langle\sigma_{x} \sigma_{y}\right\rangle_{G}}{\left\langle\sigma_{A}\right\rangle_{G}^{+}},
$$


where the event on the left denotes the fact that $x$ and $y$ are connected by a path $x=$ $x_{0} \sim \ldots \sim x_{m}=y$ of neighboring vertices of $G$ such that $\left(\mathbf{n}_{1}+\mathbf{n}_{2}\right)\left(x_{i}, x_{i+1}\right)>0$ for every $0 \leq i<m$. Sometimes, we will consider two sets $X$ and $Y$ instead of $x$ and $y$. By this, we mean that some vertex in $X$ is connected to some vertex in $Y$.

A special case of this relation consists in choosing $A=\{x, y\}$, which gives

$$
\left\langle\sigma_{x} \sigma_{y}\right\rangle_{G, \beta}=\left\langle\sigma_{x} \sigma_{y}\right\rangle_{G, \beta}^{+} \mathbb{P}_{G^{+}, G}^{\{x, y\}, \emptyset}\left[x \underset{G}{\stackrel{\mathbf{n}_{1}+\mathbf{n}_{2}}{\rightleftarrows}} y\right] .
$$

We conclude the section by proving Lemma 1.2.

Proof of Lemma 1.2. Let $G$ be a finite subgraph of $\mathbb{Z}^{d}$. For $S \subset V^{+}$, let $\mathcal{C}_{\mathbf{n}}(S)$ denote the set of all vertices in $G^{+}$which are connected to $S$ by $\mathbf{n}$. Since $A$ and $B$ are disjoint, the switching lemma implies that

$$
\left\langle\sigma_{A} \sigma_{B}\right\rangle_{G, \beta}^{+}-\left\langle\sigma_{A}\right\rangle_{G, \beta}^{+}\left\langle\sigma_{B}\right\rangle_{G, \beta}^{+}=\left\langle\sigma_{A \cup B}\right\rangle_{G, \beta}^{+} \mathbb{P}_{G^{+}, G^{+}}^{A \cup B, \emptyset}\left[1-\mathbb{I}_{\mathcal{F}_{B}}\right],
$$

where $\mathcal{F}_{B}$, when $|B|$ is even, is the event that every connected component of $\mathbf{n}_{1}+\mathbf{n}_{2}$ contains an even number of vertices from $B$. When $|B|$ is odd, $\mathcal{F}_{B}$ is the event that every connected component of $\mathbf{n}_{1}+\mathbf{n}_{2}$ contains an even number of vertices from $B \cup\{\mathfrak{g}\}$. Hence, to prove the lemma it suffices to demonstrate the inequality

$$
\begin{aligned}
\sum_{\substack{\partial \mathbf{n}_{1} \cap V=A \cup B \\
\partial \mathbf{n}_{2}=\emptyset}} \omega_{\beta}\left(\mathbf{n}_{1}\right) \omega_{\beta}\left(\mathbf{n}_{2}\right) \mathbb{I}\left[\mathbf{n}_{1}+\mathbf{n}_{2} \notin \mathcal{F}_{B}\right] \\
\leq \sum_{\substack{A^{\prime} \subset A \\
\left|A^{\prime}\right| \text { is odd }}} \sum_{\substack{B^{\prime} \subset B \\
\left|B^{\prime}\right| \text { is odd }}} \sum_{\substack{a \in A^{\prime} \\
b \in B^{\prime}}} \sum_{\substack{\partial \mathbf{n}_{1}=\{a, b\} \\
\partial \mathbf{n}_{2}=\emptyset}} \omega_{\beta}\left(\mathbf{n}_{1}\right) \omega_{\beta}\left(\mathbf{n}_{2}\right) \mathbb{I}\left[\mathbf{n}_{1}+\mathbf{n}_{2} \notin \mathcal{F}_{\{b\}]}\right] .
\end{aligned}
$$

To demonstrate (2.14), first notice that if $\mathbf{n}_{1}+\mathbf{n}_{2} \notin \mathcal{F}_{B}$, then there should be a connected component of $\mathbf{n}_{1}+\mathbf{n}_{2}$ whose intersections with $A$ and $B$ are two sets $A^{\prime}$ and $B^{\prime}$ of odd cardinality, and furthermore this component should not contain $\mathfrak{g}$. The latter assumption is valid since if all such components contained $\mathfrak{g}$, then inevitably $\mathbf{n}_{1}+\mathbf{n}_{2} \in \mathcal{F}_{B}$. Therefore we have

$$
\begin{aligned}
& \sum_{\substack{\partial \mathbf{n}_{1}=A \cup B \\
\partial \mathbf{n}_{2}=\emptyset}} \omega_{\beta}\left(\mathbf{n}_{1}\right) \omega_{\beta}\left(\mathbf{n}_{2}\right) \mathbb{I}\left[\mathbf{n}_{1}+\mathbf{n}_{2} \notin \mathcal{F}_{B}\right] \\
& \leq \sum_{\substack{A^{\prime} \subset A \\
\left|A^{\prime}\right| \text { is odd }}} \sum_{\substack{B^{\prime} \subset B \\
\left|B^{\prime}\right| \text { is odd }}} \sum_{\substack{\partial \mathbf{n}_{1}=A \cup B \\
\partial \mathbf{n}_{2}=\emptyset}} \omega_{\beta}\left(\mathbf{n}_{1}\right) \omega_{\beta}\left(\mathbf{n}_{2}\right) \mathbb{I} \\
& {\left[\mathcal{C}_{\mathbf{n}_{1}+\mathbf{n}_{2}}\left(A^{\prime}\right) \cap(A \cup B \cup\{\mathfrak{g}\})=A^{\prime} \cup B^{\prime}\right] .}
\end{aligned}
$$

We can write the third summation on the right hand side of the above display as

$$
\sum_{S \in \mathbf{S}} \sum_{\substack{\mathbf{n}_{1}, \mathbf{n}_{2} \in \mathbb{N}^{E} S \\ \partial \mathbf{n}_{1}=A^{\prime} \\ \partial \mathbf{n}_{2}=B^{\prime}}} \omega_{\beta}\left(\mathbf{n}_{1}\right) \omega_{\beta}\left(\mathbf{n}_{2}\right) \mathbb{I}\left[\mathcal{C}_{\mathbf{n}_{1}+\mathbf{n}_{2}}\left(A^{\prime}\right)=S\right]
$$




$$
\times\left(\left\langle\sigma_{A \cup B \backslash\left(A^{\prime} \cup B^{\prime}\right)}\right\rangle_{G \backslash S} \sum_{\substack{\mathbf{n}_{1}, \mathbf{n}_{2} \in \mathbb{N} E \backslash E_{S} \\ \partial \mathbf{n}_{1}=\emptyset \\ \partial \mathbf{n}_{2}=\emptyset}} \omega_{\beta}\left(\mathbf{n}_{1}\right) \omega_{\beta}\left(\mathbf{n}_{2}\right)\right)
$$

where $\mathbf{S}$ is the set of all $S \subset V^{+}$satisfying $S \cap(A \cup B \cup\{\mathfrak{g}\})=A^{\prime} \cup B^{\prime}$, and $E_{S}$ is the set of edges with at least one vertex in $S$. But this is bounded by

$$
\sum_{\substack{\partial \mathbf{n}_{1}=A^{\prime} \\ \partial \mathbf{n}_{2}=B^{\prime}}} \omega_{\beta}\left(\mathbf{n}_{1}\right) \omega_{\beta}\left(\mathbf{n}_{2}\right) \mathbb{I}\left[\mathfrak{g} \notin \mathcal{C}_{\mathbf{n}_{1}+\mathbf{n}_{2}}\left(A^{\prime}\right)=\mathcal{C}_{\mathbf{n}_{1}+\mathbf{n}_{2}}\left(B^{\prime}\right)\right]
$$

plugging which into (2.15), we get

$$
\begin{aligned}
& \sum_{\substack{\partial \mathbf{n}_{1}=A \cup B \\
\partial \mathbf{n}_{2}=\emptyset}} \omega_{\beta}\left(\mathbf{n}_{1}\right) \omega_{\beta}\left(\mathbf{n}_{2}\right) \mathbb{I}\left[\mathbf{n}_{1}+\mathbf{n}_{2} \notin \mathcal{F}_{B}\right] \\
& \quad \leq \sum_{\substack{A^{\prime} \subset A \\
\left|A^{\prime}\right| \text { is odd }}} \sum_{\substack{B^{\prime} \subset B \\
\left|B^{\prime}\right| \text { is odd }}} \sum_{\substack{\partial \mathbf{n}_{1}=A^{\prime} \\
\partial \mathbf{n}_{2}=B^{\prime}}} \omega_{\beta}\left(\mathbf{n}_{1}\right) \omega_{\beta}\left(\mathbf{n}_{2}\right) \mathbb{I}\left[\mathfrak{g} \notin \mathcal{C}_{\mathbf{n}_{1}+\mathbf{n}_{2}}\left(A^{\prime}\right)=\mathcal{C}_{\mathbf{n}_{1}+\mathbf{n}_{2}}\left(B^{\prime}\right)\right] .
\end{aligned}
$$

Now, for every $A^{\prime} \subset A$ and $B^{\prime} \subset B$,

$$
\begin{aligned}
& \sum_{\substack{\partial \mathbf{n}_{1}=A^{\prime} \cup B^{\prime} \\
\partial \mathbf{n}_{2}=\emptyset}} \omega_{\beta}\left(\mathbf{n}_{1}\right) \omega_{\beta}\left(\mathbf{n}_{2}\right) \mathbb{I}\left[\mathfrak{g} \notin \mathcal{C}_{\mathbf{n}_{1}+\mathbf{n}_{2}}\left(A^{\prime}\right)=\mathcal{C}_{\mathbf{n}_{1}+\mathbf{n}_{2}}\left(B^{\prime}\right)\right] \\
& \leq \sum_{\substack{\partial \mathbf{n}_{1}=A^{\prime} \cup B^{\prime} \\
\partial \mathbf{n}_{2}=\emptyset}} \omega_{\beta}\left(\mathbf{n}_{1}\right) \omega_{\beta}\left(\mathbf{n}_{2}\right) \mathbb{I}\left[\mathfrak{g} \notin \mathcal{C}_{\mathbf{n}_{1}+\mathbf{n}_{2}}\left(A^{\prime}\right) \cup \mathcal{C}_{\mathbf{n}_{1}+\mathbf{n}_{2}}\left(B^{\prime}\right)\right] \\
& \leq \sum_{\substack { S \in \mathbf{S}^{\prime} \\
\begin{subarray}{c}{\mathbf{n}_{1}, \mathbf{n}_{2} \in \mathbb{N}^{2} E_{S} \\
\partial \mathbf{n}_{1}=\emptyset \\
\partial \mathbf{n}_{2}=\emptyset{ S \in \mathbf { S } ^ { \prime } \\
\begin{subarray} { c } { \mathbf { n } _ { 1 } , \mathbf { n } _ { 2 } \in \mathbb { N } ^ { 2 } E _ { S } \\
\partial \mathbf { n } _ { 1 } = \emptyset \\
\partial \mathbf { n } _ { 2 } = \emptyset } }\end{subarray}} \omega_{\beta}\left(\mathbf{n}_{1}\right) \omega_{\beta}\left(\mathbf{n}_{2}\right) \mathbb{I}\left[\mathcal{C}_{\mathbf{n}_{1}+\mathbf{n}_{2}}(\mathfrak{g})=S\right]\left\langle\sigma_{A^{\prime} \cup B^{\prime}}\right\rangle_{G \backslash S} \\
& \sum_{\substack{\mathbf{n}_{1}, \mathbf{n}_{2} \in \mathbb{N}^{E \backslash E} E_{S} \\
\partial \mathbf{n}_{1}=\emptyset \\
\partial \mathbf{n}_{2}=\emptyset}} \omega_{\beta}\left(\mathbf{n}_{1}\right) \omega_{\beta}\left(\mathbf{n}_{2}\right),
\end{aligned}
$$

where $\mathbf{S}^{\prime}$ is the set of all $S \subset V^{+}$such that $S \cap\left(A^{\prime} \cup B^{\prime} \cup\{\mathfrak{g}\}\right)=\{\mathfrak{g}\}$. Since $A^{\prime}$ and $B^{\prime}$ are sets of odd cardinality, one can use the correlation inequality

$$
\left\langle\sigma_{A^{\prime} \cup B^{\prime}}\right\rangle_{G \backslash S} \leq \sum_{\substack{a \in A^{\prime} \\ b \in B^{\prime}}}\left\langle\sigma_{a} \sigma_{b}\right\rangle_{G \backslash S}
$$

coming from the Edwards-Sokal coupling and the fact that $\mathcal{F}_{A^{\prime} \cup B^{\prime}}$ is included in the event that some $a \in A^{\prime}$ is connected to some $b \in B^{\prime}$. Substituting the above inequality in (2.16) and resuming over $S \in \mathbf{S}^{\prime}$ gives us 


$$
\begin{aligned}
\sum_{\substack{\partial \mathbf{n}_{1}=A^{\prime} \\
\partial \mathbf{n}_{2}=B^{\prime}}} \omega_{\beta}\left(\mathbf{n}_{1}\right) \omega_{\beta}\left(\mathbf{n}_{2}\right) \mathbb{I}\left[\mathfrak{g} \notin \mathcal{C}_{\mathbf{n}_{1}+\mathbf{n}_{2}}\left(A^{\prime}\right)=\mathcal{C}_{\mathbf{n}_{1}+\mathbf{n}_{2}}\left(B^{\prime}\right)\right] \\
\leq \sum_{\substack{a \in A^{\prime} \\
b \in B^{\prime}}} \sum_{\substack{\partial \mathbf{n}_{1}=\{a, b\} \\
\partial \mathbf{n}_{2}=\emptyset}} \omega_{\beta}\left(\mathbf{n}_{1}\right) \omega_{\beta}\left(\mathbf{n}_{2}\right) \mathbb{I}\left[\mathfrak{g} \notin \mathcal{C}_{\mathbf{n}_{1}+\mathbf{n}_{2}}(a)=\mathcal{C}_{\mathbf{n}_{1}+\mathbf{n}_{2}}(b)\right] .
\end{aligned}
$$

Summing over $A^{\prime}$ and $B^{\prime}$ gives us (2.14) and concludes the proof.

In the rest of the article, $p$ and $\beta$ are fixed so that $1-p=e^{-2 \beta}$ and dropped from the notation.

\section{Applications of Pisztora's Coarse-Grain Approach}

The next subsection introduces the notion of good blocks. We then use a renormalization scheme to deduce that all big boxes are connected in FK-Ising. The last subsection derives Theorem 1.3 from Proposition 1.5.

3.1. Blocks and good blocks. For $k \geq 1$ and a set $S$, introduce the set $\mathcal{B}_{k}(S)$ of boxes $\Lambda_{k}(x) \subset S$ with $x \in k \mathbb{Z}^{d}$. From now on, we call an element of $\mathcal{B}_{k}(S)$ a block and often identify it with the set of its edges. Call a block $\mathbf{B} \operatorname{good}$ in $\omega$ if

(a) $\omega_{\mid \mathbf{B}}$ contains a cluster touching all the $2 d$ faces of $\mathbf{B}$;

(b) Any open path of length $k$ in $\mathbf{B}$ is included in this cluster.

Boxes of this type were used by Pisztora to derive surface order large deviation estimates for the Ising, Potts and FK-percolation models. While the notion of good box there is slightly different, we refer to the definition of $U$ before [Pis96, Theorem 3.1] for a stronger notion than the notion above. The papers [Pis96] and [Bod05] together imply, for every $p>p_{c}$, the existence of $c>0$ such that for every $k$ and every boundary condition $\xi$,

$$
\phi_{\Lambda_{2 k}}^{\xi}\left[\Lambda_{k} \text { is good }\right]>1-\mathrm{e}^{-c k}
$$

Lemma 3.1. For every $p>p_{c}$, there exists $c>0$ such that for every $n \leq N \leq e^{n^{\alpha}}$ (with $\alpha<d-1$ ) and every $x, y \in \mathbb{Z}^{d}$ such that $\Lambda_{n}(x), \Lambda_{n}(y) \subset \Lambda_{N}$,

$$
\phi_{\Lambda_{N}}^{0}\left[\Lambda_{n}(x) \longleftrightarrow \Lambda_{n}(y)\right] \geq 1-\exp \left(-c n^{d-1}\right) .
$$

Proof. Fix $p<1$. Choose $\varepsilon>0$ small enough (see later) and $k$ large enough that

$$
\phi_{\Lambda_{2 k}}^{\xi}\left[\Lambda_{k} \text { is good }\right]>1-\varepsilon
$$

for every boundary condition $\xi$. Define a site percolation $\eta$ on $\mathcal{B}_{k}\left(\Lambda_{N}\right)$ by saying that $\mathbf{B}$ is open if it is good, and closed otherwise. For a box $\mathbf{B}$, define $M(\mathbf{B})$ to be the set of all the boxes in $\mathcal{B}_{k}\left(\Lambda_{N}\right)$ whose centers are at a $\ell^{\infty}$ distance at most $3 k$ of the center of $\mathbf{B}$. Note that,

$$
\phi_{\Lambda_{N}}^{0}\left[\eta_{\mathbf{B}} \mid \eta_{\left.\left.\right|_{M(\mathbf{B})^{c}}\right] \geq 1-\varepsilon}\right.
$$


One deduces from [LSS97] that the process $\eta$ dominates a Bernoulli percolation $\tilde{\eta}$ with parameter $p$ provided that $\varepsilon$ is small enough.

A result of Deuschel and Pisztora [DP96] shows that for $p$ close enough to 1 and $n \geq$ 1, the probability that, for a fixed box $\Lambda_{n}(x)$, there exists an open cluster in $\tilde{\eta}$ containing more than three fourths of the blocks in $\mathcal{B}_{k}\left(\Lambda_{n}(x)\right)$ is larger than $1-\exp \left[-2 c n^{d-1}\right]$. The domination of $\tilde{\eta}$ by $\eta$ together with a union bound shows that this cluster also exists in $\eta$. Therefore, with probability

$$
1-\left|\Lambda_{N}\right| \exp \left(-2 c n^{d-1}\right) \geq 1-\left|\Lambda_{\exp \left(n^{\alpha}\right)}\right| \exp \left(-2 c n^{d-1}\right) \geq 1-\exp \left(-c n^{d-1}\right)
$$

(for $n$ large enough), every box $\Lambda_{n}(x)$ in $\Lambda_{N}$ satisfies that there exists an open cluster in $\eta$ containing more than half the blocks contained in $\mathcal{B}_{k}\left(\Lambda_{n}(x)\right)$.

As a immediate consequence, on this event, all these clusters [meaning for every $\Lambda_{n}(x)$ ] must in fact be the same cluster. In particular, there exist paths of pairwise neighboring good blocks going between any two boxes of size $n$ in $\Lambda_{N}$. Property (a) of a good block implies that there must be an open path of length at least $k$ in the common region between two neighboring good blocks. Property (b), on the other hand, implies that such a path must belong to the largest clusters of each of these blocks, i.e. the largest clusters of these blocks are connected. Together these give us that there exists an open path in $\omega$ between any two boxes of size $n$.

Remark 3.2. The result of [LSS97] is quantitative. Since the probability of being good is larger than $1-\mathrm{e}^{-c k}$, the process $\eta$ dominates a Bernoulli percolation of parameter $p$ equal to $1-\mathrm{e}^{-c^{\prime} k}$ for some $c^{\prime}=c^{\prime}(c)>0$ independent of $k$.

3.2. From Proposition 1.5 to Theorem 1.3. We now prove Theorem 1.3 using Proposition 1.5. Fix some boundary conditions $\xi$. We construct a coupling $\Phi_{\Lambda_{n}}^{\xi, 1}$, on pairs of configurations $\left(\omega^{\xi}, \omega^{1}\right)$ with $\phi_{\Lambda_{n}}^{\xi}$ being the law of the first marginal and $\phi_{\Lambda_{n}}^{1}$ the one of the second marginal. Call a block $\mathbf{B} \in \mathcal{B}_{k}\left(\Lambda_{n}\right)$ very good in $\left(\omega^{\xi}, \omega^{1}\right)$ if it is good in $\omega_{\mid \mathbf{B}}^{\xi}$ and $\omega_{\mid \mathbf{B}}^{\xi}=\omega_{\mid \mathbf{B}}^{1}$.

We construct algorithmically a coupling $\Phi_{\Lambda_{n}}^{\xi, 1}$ block by block following steps indexed by $t$. We assume that $k$ divides $n$ (the construction can be adapted in a trivial fashion to the general case). Below, $C_{t}$ will denote the set of edges $e$ for which $\left(\omega_{e}^{\xi}, \omega_{e}^{1}\right)$ is sampled before step $t$. The set $A_{t}$ is the set of blocks that have been sampled up to time $t$. Set

$$
\left\{\begin{array}{l}
A_{0}:=\emptyset \\
B_{0}:=\left\{\mathbf{B} \in \mathcal{B}_{k}\left(\Lambda_{n}\right), \mathbf{B} \cap\left(\Lambda_{n} \backslash \Lambda_{n-1}\right) \neq \emptyset\right\}, \\
C_{0}:=\emptyset
\end{array}\right.
$$

(The set $B_{0}$ corresponds to blocks adjacent to the boundary of $\Lambda_{n}$.) At Step $t$, the algorithm proceeds as follows:

- If $B_{t}=\emptyset$, sample $\omega_{\mid E_{n} \backslash C_{t}}^{\xi}=\omega_{\mid E_{n} \backslash C_{t}}^{1}$ according to the measure $\phi_{\Lambda_{n}}^{\xi}\left(. \mid \omega_{\mid C_{t}}^{\xi}\right)$ and terminate the algorithm.

- If $B_{t} \neq \emptyset$, choose a block $\mathbf{B} \in B_{t}$ and define $D_{t}:=\mathbf{B} \backslash C_{t}$. Then, sample $\omega_{\mid D_{t}}^{\xi} \leq \omega_{\mid D_{t}}^{1}$ such that $\omega_{\mid D_{t}}^{\xi}$ has the law $\phi_{\Lambda_{n}}^{\xi}\left[\cdot\left|D_{t}\right| \omega_{\mid C_{t}}^{\xi}\right]$ and $\omega_{\mid D_{t}}^{1}$ the law $\phi_{\Lambda_{n}}^{1}\left[\cdot\left|D_{t}\right| \omega_{\mid C_{t}}^{1}\right]$. Finally, if 
$N(\mathbf{B})$ denotes the set of blocks in $\mathcal{B}_{k}\left(\Lambda_{n}\right)$ that intersect $\mathbf{B}$, set

$$
\left\{\begin{array}{l}
A_{t+1}:=A_{t} \cup\{\mathbf{B}\}, \\
B_{t+1}:=B_{t} \backslash\{\mathbf{B}\} \text { if } \mathbf{B} \text { is very good and } B_{t} \cup N(\mathbf{B}) \backslash A_{t+1} \text { otherwise, } \\
C_{t+1}:=C_{t} \cup D_{t} .
\end{array}\right.
$$

When the algorithm terminates, set $T$ for the terminal time and return $\left(\omega^{\xi}, \omega^{1}\right)$.

Lemma 3.3. The measure $\Phi_{\Lambda_{n}}^{\xi, 1}$ satisfies that

- $\omega^{\xi}$ has law $\phi_{\Lambda_{n}}^{\xi}$ and $\omega^{1}$ has law $\phi_{\Lambda_{n}}^{1}$,

- $\omega^{\xi} \leq \omega^{1}$,

- $\omega_{e}^{\xi}=\omega_{e}^{1}$ if $e \notin C_{T}$.

Proof. To prove this lemma, it suffices to prove that the boundary conditions induced on $E_{n} \backslash C_{T}$ by $\omega_{\mid C_{T}}^{\xi}$ and $\omega_{\mid C_{T}}^{1}$ are the same. Indeed, the three items follow readily from this observation and the joint spatial Markov property. Since $\omega_{\mid C_{T}}^{\xi} \leq \omega_{\mid C_{T}}^{1}$, it suffices to prove that for every vertices $x$ and $y$ on the boundary of $E_{n} \backslash C_{T}$,

$$
x \stackrel{\omega_{\mid C_{T}}^{1}}{\longleftrightarrow} y \Longrightarrow x \stackrel{\omega_{\mid C_{T}}^{\xi}}{\longleftrightarrow} y .
$$

If $C_{T}=E_{n}$ there is nothing to prove. If $C_{T} \neq E_{n}$, let

$$
Y:=\mathcal{B}_{k}\left(\Lambda_{n}\right) \backslash A_{T} \quad \text { and } \quad Z:=\left\{\mathbf{B} \in A_{T} \text { intersecting a block in } Y\right\},
$$

where below we also identify $Z$ with the set of edges in its blocks. The fact that $B_{T}=\emptyset$ implies that $\omega^{\xi}$ and $\omega^{1}$ coincide on $Z$ and every $\mathbf{B} \in Z$ is very good for $\omega^{1}$ and $\omega^{\xi}$. Now, for every connected component $C$ of $C_{T}$, the set of blocks in $Z$ that intersect $C$ are connected in the following sense: every two such blocks $\mathbf{B}$ and $\mathbf{B}^{\prime}$ are connected by a sequence of blocks $\mathbf{B}=\mathbf{B}_{1}, \ldots, \mathbf{B}_{s}=\mathbf{B}^{\prime}$ of $Z$ such that $\mathbf{B}_{i} \cap \mathbf{B}_{i+1} \neq \varnothing$ for every $1 \leq i<s$. The discussion in the proof of Lemma 3.1 implies that all the big clusters in these blocks are connected to each other inside $Z$. Thus, if two vertices $x$ and $y$ on the boundary of $C_{T}$ are connected in $\omega_{\mid C_{T}}^{\xi}$, then they already are in $\omega_{\mid Z}^{\xi}=\omega_{\mid Z}^{1}$. This proves (3.3) and therefore concludes the proof.

Proof of Theorem 1.3. Let $D$ be the number of blocks in $M(\mathbf{B})$ (recall the definition of $M(\mathbf{B})$ from the proof of Lemma 3.1 and observe that the choice of $\mathbf{B}$ is irrelevant here), and choose $\varepsilon$ so that $2 D \varepsilon<1 / e$. By Proposition 1.5, pick $k$ large enough that

$$
\sum_{e \in \Lambda_{k}} \phi_{\Lambda_{2 k}}^{1}\left[\omega_{e}\right]-\phi_{\Lambda_{2 k}}^{0}\left[\omega_{e}\right] \leq\left|\Lambda_{k}\right| e^{-c(\log k)^{1+c}} \leq \varepsilon .
$$

Also, assume that $k$ is chosen large enough that the probability of being good is larger than $1-\varepsilon$ [this is doable by using (3.1)].

By the Markov property (2.1), (1.7) follows from the next claim: for every boundary condition $\xi$ on $\mathbb{Z}^{d}$ and every event $A$ depending on edges in $E_{n / 2}$ only,

$$
\left|\phi_{\Lambda_{n}}^{\xi}[A]-\phi_{\Lambda_{n}}^{1}[A]\right| \leq \Phi^{\xi, 1}\left[C_{T} \cap E_{n / 2} \neq \emptyset\right] \leq \exp [-c n] .
$$

The first inequality follows directly from the third item of Lemma 3.3. We therefore focus on the second one. Assume that $C_{T} \cap E_{n / 2} \neq \emptyset$. Then, there must exist a sequence 
of times $t_{1}<t_{2}<\cdots<t_{s}$ with $s \geq n /(16 k)$ such that the blocks $\mathbf{B}_{1}, \ldots, \mathbf{B}_{s}$ used by the algorithm constructing $\Phi_{\Lambda_{n}}^{\xi, 1}$ at times $t_{1}, \ldots, t_{s}$ are disjoint, not very good, and such that $\mathbf{B}_{i+1} \in M\left(\mathbf{B}_{i}\right)$ for every $1 \leq i<s$. The choice of $k$ immediately implies that conditioned on $\omega^{\xi}$ and $\omega^{1}$ in the blocks $\mathbf{B}_{1}, \ldots, \mathbf{B}_{i}$, the block $\mathbf{B}_{i+1}$ is not very good with probability smaller than or equal to $2 \varepsilon$. We deduce that

$$
\Phi_{\Lambda_{n}}^{\xi, 1}\left[C_{T} \cap E_{n / 2} \neq \emptyset\right] \leq(D 2 \varepsilon)^{n /(16 k)} .
$$

The choice of $\varepsilon$ proves (3.4) with $c=1 /(16 k)$.

\section{Proof of Proposition 1.5}

The proof of Proposition 1.5 can be decomposed into several steps. We first prove a very weak mixing property (where the Radon-Nikodym derivative is bounded from above by $\exp \left(c n^{d-1}\right)$. We then use this property to show Proposition 1.5, but basing our study on two technical lemmata whose proofs are postponed to the next section.

4.1. Connection probability between boxes for double random current. The main result of this section is the proposition below. The proof relies on a number of properties of the random current, combined with a mixing property for the Fortuin-Kasteleyn percolation.

Proposition 4.1. Fix $\beta>\beta_{c}$. There exists $c>0$ such that for every $N \geq 2 n$, every $x, y, w, z \in \Lambda_{N}$ with $w, z \notin \Lambda_{n}(x) \cup \Lambda_{n}(y) \subset \Lambda_{N}$, we have

$$
\mathbb{P}_{\Lambda_{N}^{+}, \Lambda_{N}}^{\{w, z\}, \emptyset}\left[\Lambda_{n}(x) \underset{\Lambda_{N}}{\stackrel{\mathbf{n}_{1}+\mathbf{n}_{2}}{\longrightarrow}} \Lambda_{n}(y)\right] \geq 1-\mathrm{e}^{-c n^{d-1}} .
$$

In the whole section, we will use the following notation. Without loss of generality, we can assume that $n$ is even. Assume that $\Lambda_{n}(x) \cap \Lambda_{n}(y)=\emptyset$. Construct the graphs $\Lambda_{N}^{\bullet \bullet}:=\Lambda_{N}^{\bullet \bullet}(x, y, n)$ and $\left(\Lambda_{N}^{\bullet \bullet}\right)^{+}:=\left(\Lambda_{N}^{\bullet \bullet}(x, y, n)\right)^{+}$obtained from $\Lambda_{N}$ and $\Lambda_{N}^{+}$by replacing $\Lambda_{n / 2}(x)$ and $\Lambda_{n / 2}(y)$ by two vertices $\mathbf{x}$ and $\mathbf{y}$, which are connected to each vertex outside $\Lambda_{n / 2}(x) \cup \Lambda_{n / 2}(y)$ by the number of edges between this vertex and $\Lambda_{n / 2}(x)$ (resp. $\Lambda_{n / 2}(y)$ ). Similarly, one defines $\Lambda_{n}^{\bullet}(x)$ to be the graph obtained from $\Lambda_{n}(x)$ by merging all the vertices in $\Lambda_{n / 2}(x)$ following the same procedure as for $\Lambda_{N}^{\bullet \bullet}$. Note that the FK-Ising and Ising models on those graphs can be seen as models on the original graphs, for which edges $e$ in $\Lambda_{n / 2}(x) \cup \Lambda_{n / 2}(y)$ have $p_{e}=1$ (for the FK-Ising) and have infinite coupling constants (for the Ising model). This observation is useful to keep in mind when applying (2.8) for instance.

Proof. If $\Lambda_{n}(x) \cap \Lambda_{n}(y) \neq \emptyset$, one does not need to do anything. We therefore assume that $\Lambda_{n}(x) \cap \Lambda_{n}(y)=\varnothing$ and consider the graph $\Lambda_{N}^{\bullet \bullet}$ defined above. Equation (2.12) implies that

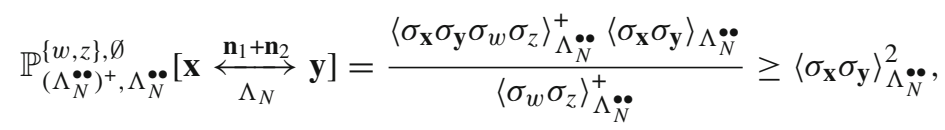

where in the second inequality we used Griffiths' inequalities (2.7) and (2.8). The Edwards-Sokal coupling (2.5) and the FKG inequality (2.3) (the measure on $\Lambda_{N}^{\bullet \bullet}$ can 
be understood as the measure on $\Lambda_{N}$ with edges in $\Lambda_{n / 2}(x) \cup \Lambda_{n / 2}(y)$ conditioned to be open) imply that

$$
\left\langle\sigma_{\mathbf{x}} \sigma_{\mathbf{y}}\right\rangle_{\Lambda_{N}}=\phi_{\Lambda_{N}^{\bullet}}[\mathbf{x} \leftrightarrow \mathbf{y}] \geq \phi_{\Lambda_{N}}\left[\Lambda_{n / 2}(x) \leftrightarrow \Lambda_{n / 2}(y)\right] \geq 1-\exp \left(-c n^{d-1}\right),(4
$$

where the last inequality follows from Lemma 3.1.

Assume for a moment that for every $\varepsilon>0$, one can choose $n$ large enough that for every choice of $N$ and $x, y$, for every event $\mathcal{E}$ depending on $\left(\mathbf{n}_{1}, \mathbf{n}_{2}\right)$ on edges in $E:=E_{N} \backslash\left(E_{n}(x) \cup E_{n}(y)\right)$ only, we have that

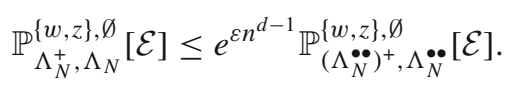

Then, one would deduce from plugging the estimate (4.2) into (4.1), and then using (4.3) (together with the fact that if $\mathbf{x}$ is connected to $\mathbf{y}$, then $\Lambda_{n}(x)$ is connected to $\Lambda_{n}(y)$ ), that

$$
\mathbb{P}_{\Lambda_{N}^{+}, \Lambda_{N}}^{\{w, z\}, \emptyset}\left[\Lambda_{n}(x) \underset{\Lambda_{N}}{\stackrel{\mathbf{n}_{1}+\mathbf{n}_{2}}{\longrightarrow}} \Lambda_{n}(y)\right] \leq e^{\varepsilon n^{d-1}} \mathbb{P}_{\left(\Lambda_{N}^{\circ}\right)^{+}, \Lambda_{N}^{\circ}}^{\{w, z\}, \emptyset}\left[\Lambda_{n}(x) \underset{\Lambda_{N}}{\stackrel{\mathbf{n}_{1}+\mathbf{n}_{2}}{\longrightarrow}} \Lambda_{n}(y)\right] \leq e^{(\varepsilon-2 c) n^{d-1}},
$$

which would conclude the proof. We therefore focus on proving (4.3). Let $\Lambda:=\Lambda_{n}(x) \cup$ $\Lambda_{n}(y)$ and consider a current $\mathbf{m} \in \mathbb{N}^{E}$ with $\partial \mathbf{m} \subset \partial \Lambda$. Let us also consider the event $\mathcal{E}_{\mathbf{m}}$ that $\mathbf{n}$ is equal to $\mathbf{m}$ on $E$. A trivial manipulation using the expression of weights for currents (in the first line) and then Griffiths inequality (2.8) in the second give that

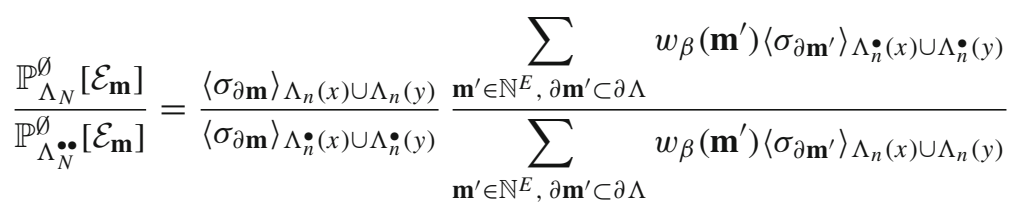

$$
\begin{aligned}
& \leq \max _{\substack{A \subset \partial \Lambda_{n}(x) \\
|A| \text { even }}} \frac{\left\langle\sigma_{A}\right\rangle_{\Lambda_{n}^{\bullet}(x)}}{\left\langle\sigma_{A}\right\rangle_{\Lambda_{n}(x)}} \times \max _{\substack{B \subset \partial \Lambda_{n}(y) \\
|B| \text { even }}} \frac{\left\langle\sigma_{B}\right\rangle_{\Lambda_{n}^{\bullet}(y)}}{\left\langle\sigma_{B}\right\rangle_{\Lambda_{n}(y)}},
\end{aligned}
$$

where the sums on the first line are on currents on $E$ (with the right sources). A similar identity can be derived for $\mathbb{P}_{\Lambda_{N}^{+}}^{\{z, w\}}$ and $\mathbb{P}_{\left(\Lambda_{N}^{\circ}\right)^{+}}^{\{z, w\}}$ instead of $\mathbb{P}_{\Lambda_{N}}^{\emptyset}$ and $\mathbb{P}_{\Lambda_{N}}^{\emptyset}$.

We deduce, by decomposing on possible values of $\left(\mathbf{n}_{1}, \mathbf{n}_{2}\right)$ on $E$, that

$$
\mathbb{P}_{\Lambda_{N}^{+}, \Lambda_{N}}^{\{w\}, \emptyset}[\mathcal{E}] \leq\left(\max _{\substack{A \subset \partial \Lambda_{n} \\|A| \text { even }}} \frac{\left\langle\sigma_{A}\right\rangle_{\Lambda_{n}^{\bullet}}}{\left\langle\sigma_{A}\right\rangle_{\Lambda_{n}}}\right)^{4} \mathbb{P}_{\left(\Lambda_{N}^{\bullet}\right)^{+}, \Lambda_{N}^{\bullet \bullet}}^{\{w, E],}
$$

so that (4.3) follows from the following lemma.

Lemma 4.2. Fix $\beta>\beta_{c}$ and $\varepsilon>0$. Then, for $n$ large enough, we have that for every $A \subset \partial \Lambda_{n}$,

$$
\left\langle\sigma_{A}\right\rangle_{\Lambda_{n}} \leq \mathrm{e}^{\varepsilon n^{d-1}}\left\langle\sigma_{A}\right\rangle_{\Lambda_{n}} .
$$


Proof. Fix $\beta>\beta_{c}$ and set $p=1-e^{-2 \beta}>p_{c}$. By (2.5), we must prove that $\phi_{\Lambda_{n}^{\bullet}}^{0}\left[\mathcal{F}_{A}\right] \leq$ $\mathrm{e}^{\varepsilon n^{d-1}} \phi_{\Lambda_{n}}^{0}\left[\mathcal{F}_{A}\right]$. Note that since $\varepsilon$ is arbitrary and since $\mathcal{F}_{A}$ occurs if all the edges on the boundary of $\Lambda_{n}$ are open (and therefore has a probability larger than $\left(\frac{p}{2-p}\right)^{d\left|\partial \Lambda_{n}\right|}$ ), it suffices to show that

$$
\phi_{\Lambda_{n}^{\bullet}}^{0}\left[\mathcal{F}_{A}\right] \leq \mathrm{e}^{-c n^{d}}+n\left(\frac{2-p}{p}\right)^{\varepsilon n^{d-1}} \phi_{\Lambda_{n}}^{0}\left[\mathcal{F}_{A}\right]
$$

We do this by constructing a coupling $\Phi$ on pairs $\left(\omega, \omega^{\bullet}\right)$ of percolation configurations on $\{0,1\}^{E_{n}}$ for which $\omega \leq \omega^{\bullet}$ have respective laws $\phi_{\Lambda_{n}}^{0}$ and $\phi_{\Lambda_{n}}^{0}$ (for consistency, we see a configuration on $\Lambda_{n}^{\bullet}$ as a configuration on $E_{n}$ for which edges in $E_{n / 2}$ are automatically open).

Consider $k$ to be fixed later and assume that $k$ divides $n / 2$ (one can trivially adapt the proof if $k$ does not). Also, set $T:=n /(4 k)$. A block $\mathbf{B}$ is called bad if it is either not good or if $\omega_{\mid \mathbf{B}} \neq \omega_{\mid \mathbf{B}}^{\bullet}$. For every $t \in[T, 2 T]$, define the three sets $C_{t}:=E_{2 k t}, D_{t}$ the set of edges in $C_{t+1} \backslash C_{t}$ within a distance of $2 k$ of a bad block included in $C_{t}$, and $D_{t}^{\prime}:=C_{t+1} \backslash\left(C_{t} \cup D_{t}\right)$; see Fig. 1 . Note that $C_{t}$ is a deterministic set, but $D_{t}$ and $D_{t}^{\prime}$ are random variables which are measurable in terms of $\left(\omega_{\mid C_{t}}, \omega_{\mid C_{t}}^{\bullet}\right)$.

Then, the coupling is constructed as follows. First, sample $\omega_{\mid E_{n / 2}}$ according to $\phi_{\Lambda_{n}}\left(\cdot \mid E_{n / 2}\right)$ (recall that the edges of $\omega^{\bullet}$ are necessarily open in $E_{n / 2}$ ). Then, for every $t \geq T$,

- Sample $\omega_{\mid D_{t}} \leq \omega_{\mid D_{t}}^{\bullet}$ according to $\phi_{\Lambda_{n}}^{0}\left({ }^{\mid}\left|D_{t}\right| \omega_{\mid C_{t}}\right)$ and $\phi_{\Lambda_{n}^{\bullet}}^{0}\left(\cdot\left|D_{t}\right| \omega_{\mid C_{t}}^{\bullet}\right)$,

- Sample $\omega_{\mid D_{t}^{\prime}} \leq \omega_{\mid D_{t}^{\prime}}^{\bullet}$ according to $\phi_{\Lambda_{n}}^{0}\left({ }^{\mid}\left|D_{t}^{\prime}\right| \omega_{\mid C_{t} \cup D_{t}}\right)$ and $\phi_{\Lambda_{n}^{*}}^{0}\left({ }^{\prime}\left|D_{t}^{\prime}\right| \omega_{\mid C_{t} \cup D_{t}}^{\bullet}\right)$.

For $t \in[T, 2 T]$, we call $\mathcal{G}_{t}$ the event that there are fewer than $\varepsilon n^{d-1}$ edges in $D_{t}$. The inequality (4.5) follows readily from the following two claims.

Claim 1. For every $t \geq T$, we have that

$$
\Phi\left[\left\{\omega^{\bullet} \in \mathcal{F}_{A}\right\} \cap \mathcal{G}_{t}\right] \leq\left(\frac{2-p}{p}\right)^{\varepsilon n^{d-1}} \Phi\left[\omega \in \mathcal{F}_{A}\right] .
$$

Claim 2. There exist $k$ and $c>0$ such that for every $n$ large enough,

$$
\Phi\left[\bigcap_{t \geq T} \mathcal{G}_{t}^{c}\right] \leq \mathrm{e}^{-c n^{d}} .
$$

To conclude, we only need to prove those claims.

Proof of Claim 1. Let $\mathcal{H}_{t}$ be the event that every edge in $D_{t}$ is open. We refer the reader to Fig. 1 for an illustration. On $\mathcal{H}_{t} \cap \mathcal{G}_{t}$, the boundary conditions on $E_{n} \backslash\left(C_{t} \cup D_{t}\right)$ induced by $\omega$ and $\omega^{\bullet}$ are the same. Since $\mathcal{H}_{t} \cap \mathcal{G}_{t}$ depends on edges in $C_{t} \cup D_{t}$ only, the joint Markov property of the coupling implies that $\omega_{\mid E_{n} \backslash\left(C_{t} \cup D_{t}\right)}=\omega_{\mid E_{n} \backslash\left(C_{t} \cup D_{t}\right)}^{\bullet}$ and $\omega^{\bullet} \in \mathcal{F}_{A}$ if and only if $\omega \in \mathcal{F}_{A}$. We deduce that

$$
\Phi\left[\left\{\omega^{\bullet} \in \mathcal{F}_{A}\right\} \cap \mathcal{H}_{t} \cap \mathcal{G}_{t}\right]=\Phi\left[\left\{\omega \in \mathcal{F}_{A}\right\} \cap \mathcal{H}_{t} \cap \mathcal{G}_{t}\right] \leq \Phi\left[\omega \in \mathcal{F}_{A}\right]
$$

Conditioned on $\left(\omega, \omega^{\bullet}\right)_{\mid C_{t}}$, the joint FKG inequality and the joint Markov property imply that

$$
\Phi\left[\left\{\omega^{\bullet} \in \mathcal{F}_{A}\right\} \cap \mathcal{H}_{t} \mid\left(\omega, \omega^{\bullet}\right)_{\mid C_{t}}\right] \geq \Phi\left[\left\{\omega^{\bullet} \in \mathcal{F}_{A}\right\} \mid\left(\omega, \omega^{\bullet}\right)_{\mid C_{t}}\right] \Phi\left[\mathcal{H}_{t} \mid\left(\omega, \omega^{\bullet}\right)_{\mid C_{t}}\right] .(4.7)
$$




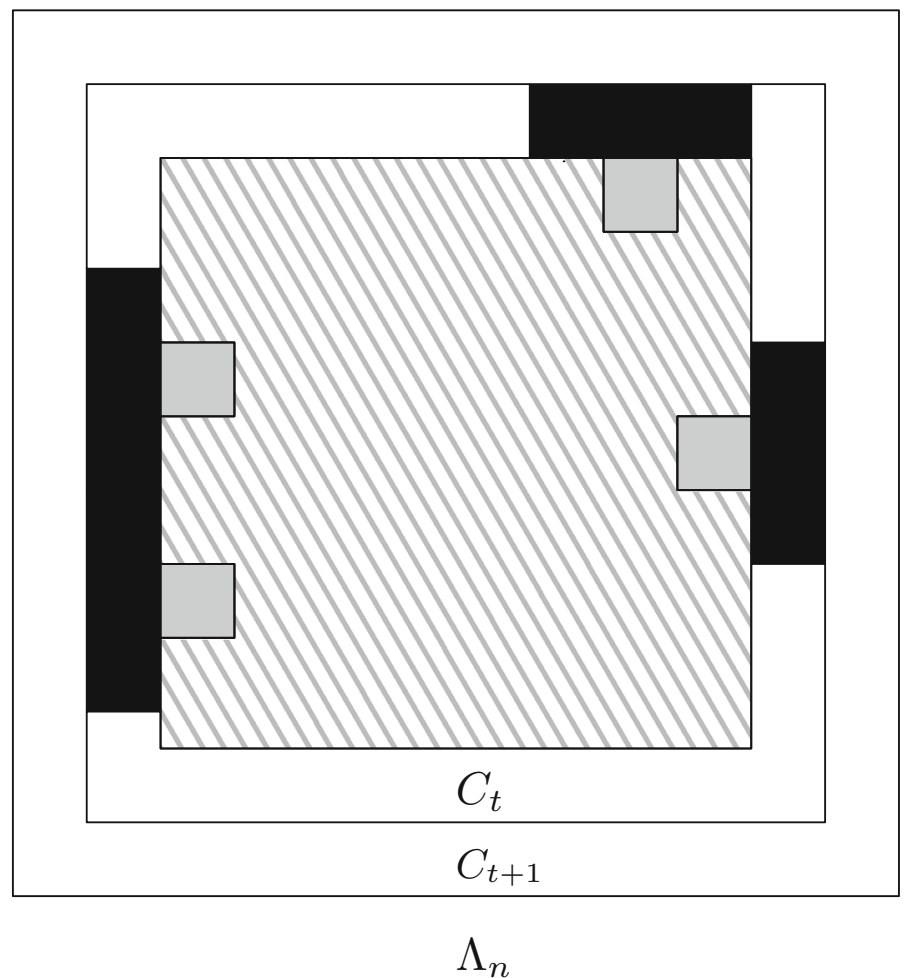

Fig. 1. A realization of the event $\mathcal{H}_{t}$. The grey boxes are the bad blocks in $C_{t}$ that are adjacent to $C_{t+1} \backslash C_{t}$. The dark rectangles represent the edges in $D_{t}$

If $\left(\omega, \omega^{\bullet}\right)_{\mid C_{t}} \in \mathcal{G}_{t}$, at most $\varepsilon n^{d-1}$ edges must be open for $\mathcal{H}_{t}$ to occur. We deduce that

$$
\Phi\left[\mathcal{H}_{t} \mid\left(\omega, \omega^{\bullet}\right)_{\mid C_{t}}\right] \geq\left(\frac{p}{2-p}\right)^{\varepsilon n^{d-1}} .
$$

Since $\mathcal{G}_{t}$ depends on edges in $C_{t}$ only, we may insert the previous estimate in (4.7) and then integrate on $\left(\omega, \omega^{\bullet}\right)_{\mid C_{t}} \in \mathcal{G}_{t}$ to obtain that

$$
\Phi\left[\left\{\omega^{\bullet} \in \mathcal{F}_{A}\right\} \cap \mathcal{H}_{t} \cap \mathcal{G}_{t}\right] \geq \Phi\left[\left\{\omega^{\bullet} \in \mathcal{F}_{A}\right\} \cap \mathcal{G}_{t}\right]\left(\frac{p}{2-p}\right)^{\varepsilon n^{d-1}} .
$$

Putting this inequality into (4.6) concludes the proof of Claim 1.

Proof of Claim 2. For no $\mathcal{G}_{t}$ to occur, there must be at least $\varepsilon n^{d-1} /\left|E_{3 k}\right| \times n /(4 k)=$ : $\varepsilon_{k}\left|E_{n}\right|$ bad blocks. As a consequence, one of the following three things must happen:

- There are more than $\left(\varepsilon_{k} / 2\right)\left|E_{n}\right|$ blocks in $\mathcal{B}_{k}\left(\Lambda_{n}\right)$ that are not good.

- The number of open edges in $\omega_{\mid E_{n} \backslash E_{n / 2}}^{\bullet}$ is larger than $\left(\phi\left[\omega_{e}\right]+\varepsilon_{k} / 4\right)\left|E_{n} \backslash E_{n / 2}\right|$.

- The number of open edges in $\omega_{\mid E_{n} \backslash E_{n / 2}}$ is smaller than $\left(\phi\left[\omega_{e}\right]-\varepsilon_{k} / 4\right)\left|E_{n} \backslash E_{n / 2}\right|$.

Note that these three events are involving either $\omega$ or $\omega^{\bullet}$, so that we can now ignore the coupling $\Phi$. We bound the probability of each one of these events separately.

For the first item, Remark 3.2 enables us to choose $k$ large enough that the process of good boxes dominates a Bernoulli percolation of parameter $p>1-\varepsilon_{k}$. We deduce 
from large deviations for iid Bernoulli variables that the probability of the event decays as $e^{-c n^{d}}$ uniformly in $n$, where $c=c(k, \varepsilon)>0$.

For the second item, the uniqueness of the infinite-volume measure $\phi$ implies that one may choose $K=K(k, \varepsilon)$ large enough that

$$
\phi_{\Lambda_{K}}^{1}\left[\sum_{e \in E_{K}} \omega_{e}\right]<\left(\phi\left[\omega_{e}\right]+\varepsilon_{k} / 4\right)\left|E_{K}\right|
$$

Now, consider a family of balls of size $K$ covering $\Lambda_{n}$ and with disjoint interiors. By sampling the FK-Ising measure $\phi_{\Lambda_{K}}^{1}$ ball by ball (here, the order in which the balls are sampled is irrelevant), the comparison between boundary conditions (2.4) enables us to compare the number of open edges in $E_{n} \backslash E_{n / 2}$ to a sum of independent random variables. The theory of large deviations implies that the probability of the second event is also bounded by $\exp \left(-c n^{d}\right)$ uniformly in $n$, where $c=c(K, k, \varepsilon)>0$.

The third item follows from the same reasoning as the second one (except it does not involve the uniqueness of the infinite-volume measure).

4.2. Proof of proposition 1.5. In the rest of the paper, for a current $\mathbf{n}$ on $\Lambda_{N}$ or $\Lambda_{N}^{+}$, we use $\mathcal{C}_{\mathbf{n}}(S)$ to denote the set of all vertices in $\Lambda_{N}$ which are connected to $S$ by $\mathbf{n}$. Notice that this is slightly different from the convention in Lemma 1.2 where we also included $\mathfrak{g}$ in $\mathcal{C}_{\mathbf{n}}(S)$.

From now on, we fix $N=N(n):=e^{n^{\alpha}}$ where $1<\alpha<d-1$, and $e=\{x, y\}$. Using (2.5) and a simple relation between the probability of $\omega_{e}=1$ and the probability that $x$ and $y$ are connected together, we find that

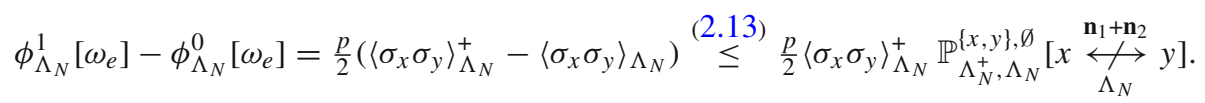

Notice that $x$ and $y$ are the sources of $\mathbf{n}_{1}$ and as such they must be connected to each other by $\mathbf{n}_{1}$ in $\Lambda_{N}^{+}$. Thus, when $x$ is not connected to $y$ in $\Lambda_{N}$, there must be a path between $x$ and $\partial \Lambda_{N}$ in $\mathcal{C}_{\mathbf{n}_{1}}(x)$ on one side, and between $y$ and $\partial \Lambda_{N}$ in $\mathcal{C}_{\mathbf{n}_{1}}(y)$ on the other side. Since $\{x, y\} \in E_{N / 4}$, the path emanating from $y$ must intersect at least $\frac{1}{2} N / n$ blocks in $\mathcal{B}_{n}\left(\Lambda_{N}\right)$. Among these blocks, there is always either $\frac{1}{4} N / n$ many intersecting $\mathcal{C}_{\mathbf{n}_{1}+\mathbf{n}_{2}}(x)$ or $\frac{1}{4} N / n$ many not intersecting it. Repeating a similar argument for the path between $x$ and $\partial \Lambda_{N}$, we obtain that if $x$ is not connected to $y$ in $\mathbf{n}_{1}+\mathbf{n}_{2}$ and we define the following events,

$$
\begin{aligned}
\mathcal{C}_{x, y}:= & \left\{\text { there exist } \frac{1}{4} N / n \text { blocks } \mathbf{B} \in \mathcal{B}_{n}\left(\Lambda_{N}\right) \text { such that } \mathcal{C}_{\mathbf{n}_{1}+\mathbf{n}_{2}}(x) \cap \mathbf{B}=\emptyset \text { and } \mathcal{C}_{\mathbf{n}_{1}}(y) \cap \mathbf{B}\right. \\
& \text { contains a vertex having at least two neighbors in } \left.\mathcal{C}_{\mathbf{n}_{1}}(y)\right\}, \\
\mathcal{B}_{x, y}:= & \text { there exist } \frac{1}{4} N / n \text { blocks } \mathbf{B} \in \mathcal{B}_{n}\left(\Lambda_{N}\right) \text { such that } \mathcal{C}_{\mathbf{n}_{1}+\mathbf{n}_{2}}(x) \cap \mathbf{B} \neq \emptyset \text { and } \mathcal{C}_{\mathbf{n}_{1}}(y) \cap \mathbf{B} \\
& \text { contains a vertex having at least two neighbors in } \left.\mathcal{C}_{\mathbf{n}_{1}}(y)\right\},
\end{aligned}
$$

then, either $\mathcal{B}_{x, y}, \mathcal{B}_{y, x}$ or $\mathcal{C}:=\mathcal{C}_{x, y} \cap \mathcal{C}_{y, x}$ must occur.

Therefore, it suffices to show that the intersection of the event on the right hand side of (4.8) with either $\mathcal{C}, \mathcal{B}_{x, y}$ or $\mathcal{B}_{y, x}$ has very small probability. This is the subject of the two lemmata below which are proved in the next section. 
Lemma 4.3. There exists $c>0$ such that for every $n$ large enough such that $\{x, y\} \in$ $E_{N / 4}$

$$
\mathbb{P}_{\Lambda_{N}, \Lambda_{N}}^{\{x, y\}, \emptyset}\left[\mathcal{C} \cap\left\{x \underset{\Lambda_{N}}{\stackrel{\mathbf{n}_{1}+\mathbf{n}_{2}}{\leftrightarrows}} y\right\}\right] \leq \mathrm{e}^{-c n^{d-1}} .
$$

Lemma 4.4. There exists $c>0$ such that for every $n$ large enough such that $\{x, y\} \in$ $E_{N / 4}$,

$$
\mathbb{P}_{\Lambda_{N}^{+}, \Lambda_{N}}^{\{x, y\}, \emptyset}\left[\mathcal{B}_{x, y} \cap\left\{x \underset{\Lambda_{N}}{\stackrel{\mathbf{n}_{1}+\mathbf{n}_{2}}{\longleftrightarrow}} y\right\}\right] \leq \mathrm{e}^{-c n^{d-1}}
$$

Note that the bounds given below imply Proposition 1.5 since $N=\mathrm{e}^{n^{\alpha}}$ gives that the bounds on the right are of the form $\exp \left[-(\log N)^{(d-1) / \alpha}\right]$, and that $\alpha<d-1$.

\section{Proofs of lemmata 4.3 and 4.4}

In the remainder of the paper, we will use $C$ and $c$ to denote finite, positive constants depending on at most $\beta$ and $d$. The values of these constants may vary from one line to the next. It might be helpful to think of $C$ and $c$ as large and small positive numbers respectively.

In order to prove these lemmata, we will use a multi-valued map principle.

Lemma 5.1. Consider a probability space $(\mathcal{S}, \mathfrak{P}(\mathcal{S}), \mu)$ where $\mathcal{S}$ is at most countable and $\mathfrak{P}(\mathcal{S})$ is the set of all subsets of $\mathcal{S}$. Let $A, B \subset \mathcal{S}$ and $\mathcal{R} \subset A \times B$ be a relation satisfying the following two properties:

(i) $|\mathcal{R}(s)| \geq K$ for every $s \in A$ where $\mathcal{R}(s):=\left\{s^{\prime} \in B:\left(s, s^{\prime}\right) \in \mathcal{R}\right\}$.

(ii) $\sum_{s \in \mathcal{R}^{-1}\left(s^{\prime}\right)} \mu(s) \leq k \mu\left(s^{\prime}\right)$ for every $s^{\prime} \in B$ where $\mathcal{R}^{-1}\left(s^{\prime}\right):=\left\{s \in A:\left(s, s^{\prime}\right) \in \mathcal{R}\right\}$.

Then, we have that

$$
\mu(A) \leq \frac{k}{K} \mu(B)
$$

Proof. This is a simple application of "counting in two ways":

$$
K \mu(A) \stackrel{(i)}{\leq} \sum_{\left(s, s^{\prime}\right) \in \mathcal{R}} \mu(s)=\sum_{s^{\prime} \in B} \sum_{s \in \mathcal{R}^{-1}\left(s^{\prime}\right)} \mu(s) \stackrel{(i i)}{\leq} \sum_{s^{\prime} \in B} k \mu\left(s^{\prime}\right)=k \mu(B) .
$$

Before we state the lemmata that are needed to invoke Lemma 5.1, let us discuss the motivation behind them. Let us take the example of the proof of Lemma 4.3 (Lemma 4.4 is very similar). Our goal is to apply the previous lemma for $A$ being the event under consideration in the lemma, and $B$ the full space of pairs of currents. Since a relation can be viewed as a multi-valued map, we can specify a relation on pairs of currents by describing several ways of modifying a given pair of currents $\left(\mathbf{n}_{1}, \mathbf{n}_{2}\right)$. In order to do so, we first perform the following two steps:

- Select a family $Z$ of blocks $\mathbf{B} \in \mathcal{B}_{n}\left(\Lambda_{N}\right)$ that all intersect both $\mathcal{C}_{\mathbf{n}_{1}}(y)$ and $\mathcal{C}_{\mathbf{n}_{1}+\mathbf{n}_{2}}(x)$. The number of choices for $Z$ will guarantee that $K$ is large. 
- For each block $\mathbf{B}$, change the value of $\mathbf{n}_{2}$ along the edges of a shortest path $\Pi_{\mathbf{B}}$ between $\mathcal{C}_{\mathbf{n}_{1}}(y) \cap \mathbf{B}$ and $\mathcal{C}_{\mathbf{n}_{1}+\mathbf{n}_{2}}(x) \cap \mathbf{B}$ so that these two sets become connected in $\mathbf{B}$ by the resulting pair of currents.

Let us call the new pair of currents $\left(\mathbf{n}_{1}^{\prime}, \mathbf{n}_{2}^{\prime}\right.$ ) (note that $\mathbf{n}_{1}^{\prime}=\mathbf{n}_{1}$ at this stage). We want to be able to recover $Z$ from $\left(\mathbf{n}_{1}^{\prime}, \mathbf{n}_{2}^{\prime}\right)$ to ensure a small value of $k$ in Property (ii) of Lemma 5.1 (called the reconstruction step below). Since $x$ and $y$ are not connected in $\Lambda_{N}$ by $\mathbf{n}_{1}+\mathbf{n}_{2}$, a natural guess for $Z$ would include any block $\mathbf{B}$ containing a vertex $v \in \mathcal{C}_{\mathbf{n}_{1}}(y)$ which is connected to $x$ by $\mathbf{n}_{1}^{\prime}+\mathbf{n}_{2}^{\prime}$ through a path in $\left(\Lambda_{N} \backslash \mathcal{C}_{\mathbf{n}_{1}^{\prime}}(y)\right) \cup\{v\}$. Unfortunately, this guess may not be correct because changing $\mathbf{n}_{2}$ could have created many such vertices $v$ apart from the endpoints of the paths $\Pi_{\mathbf{B}}$.

One way to address this problem is to additionally (and brutally) require that $\left(\mathbf{n}_{1}^{\prime}+\right.$ $\left.\mathbf{n}_{2}^{\prime}\right)(e)=0$ for all the edges $e$ adjacent to the paths $\Pi_{\mathbf{B}}$ except those adjacent to their endpoints, but this has the disadvantage of introducing new sources to the currents. In order to remove these sources, we change the value of $\left(\mathbf{n}_{1}^{\prime}, \mathbf{n}_{2}^{\prime}\right)$ along some new paths connecting pairs of sources. These new paths should avoid the paths $\Pi_{\mathbf{B}}$ except, possibly, their endpoints in $\mathcal{C}_{\mathbf{n}_{1}}(y)$ and $\mathcal{C}_{\mathbf{n}_{1}+\mathbf{n}_{2}}(x)$ so that we do not create any additional connections between $\mathcal{C}_{\mathbf{n}_{1}}(y)$ and $x$. Furthermore, we want to find these paths in neighborhoods of fixed radius around the paths $\Pi_{\mathbf{B}}$ to prevent that the value of $\left(\mathbf{n}_{1}, \mathbf{n}_{2}\right)$ is changed on too many edges as that would, again, give a large value of $k$ in the reconstruction step.

Our next lemma shows that it is always possible to find such paths within a graph distance of at most 2 from the paths $\Pi_{\mathbf{B}}$ for some specific choices of the latter. Due to purely technical reasons which we will leverage in our proof of Lemma 4.3, we prove this lemma for $\mathcal{C}_{\mathbf{n}_{1}+\mathbf{n}_{2}}(S)$ (with $S$ an arbitrary set) instead of $\mathcal{C}_{\mathbf{n}_{1}+\mathbf{n}_{2}}(x)$. Without loss of generality, we implicitly assume in the rest of this paper that the size parameters $N$ and $n$ are integer powers of 2. Also we will use distance for the $\ell_{\infty}$ distance and graph distance for the $\ell_{1}$ distance on $\mathbb{Z}^{d}$. We say that $A$ is connected in $B$ if any two vertices of $A$ can be connected by a path of vertices in $B$. We refer the reader to Fig. 2 for a pictorial description of Lemma 5.2.

Lemma 5.2. Let $S \subset \Lambda_{N}$ be such that $\mathcal{C}_{\mathbf{n}_{1}}(y) \cap \mathcal{C}_{\mathbf{n}_{1}+\mathbf{n}_{2}}(S)=\emptyset$. Assume that there exists $a$ block $\mathbf{B} \in \mathcal{B}_{n}\left(\Lambda_{N}\right)$ intersecting $\mathcal{C}_{\mathbf{n}_{1}+\mathbf{n}_{2}}(S)$ and containing a vertex in $\mathcal{C}_{\mathbf{n}_{1}}(y)$ with at least two neighbors in $\mathcal{C}_{\mathbf{n}_{1}}(y)$. Then, there exists a path $\Pi_{\mathbf{B}}=\Pi_{\mathbf{B}}\left(\mathbf{n}_{1}, \mathbf{n}_{2}\right)=\left(v_{0}, v_{1}, \ldots, v_{k}\right)$ satisfying that

- $v_{0} \in \mathcal{C}_{\mathbf{n}_{1}+\mathbf{n}_{2}}(S)$ is within a distance of at most $3 d n$ of the center of $\mathbf{B}$,

- $v_{k} \in \mathcal{C}_{\mathbf{n}_{1}}(y)$ is within a distance of at most $3 d n$ of the center of $\mathbf{B}$,

- $\Pi_{\mathbf{B}}$ is a shortest path between $v_{0}$ and $v_{k}$,

- For every $0<i<k, v_{i} \notin \mathcal{C}_{\mathbf{n}_{1}}(y) \cup \mathcal{C}_{\mathbf{n}_{1}+\mathbf{n}_{2}}(S)$,

- The set $T_{\mathbf{B}}=T_{\mathbf{B}}\left(\mathbf{n}_{1}, \mathbf{n}_{2}\right)$ of vertices in $\Lambda_{N} \backslash \mathcal{C}_{\mathbf{n}_{1}+\mathbf{n}_{2}}(S)$ at a graph distance exactly 1 of $\Pi_{\mathbf{B}} \backslash\left\{v_{0}\right\}$ is connected in the set $S_{\mathbf{B}}=S_{\mathbf{B}}\left(\mathbf{n}_{1}, \mathbf{n}_{2}\right)$ of vertices of $\Lambda_{N} \backslash \mathcal{C}_{\mathbf{n}_{1}+\mathbf{n}_{2}}(S)$ which are either equal to $v_{k}$ or at a graph distance 1 or 2 of $\Pi_{\mathbf{B}}$.

We will prove Lemma 5.2 (which is fairly technical but quite clear conceptually) at the end of the paper. With this lemma at our disposal, we can now formally describe the modification of $\left(\mathbf{n}_{1}, \mathbf{n}_{2}\right)$ that we discussed earlier. This is the content of the following lemma.

Remark 5.3. The requirement that the block $\mathbf{B}$ contains a vertex of $\mathcal{C}_{\mathbf{n}_{1}}(y)$ having two neighbors in $\mathcal{C}_{\mathbf{n}_{1}}(y)$ is used in this lemma and in this lemma only.

We introduce a few notation. From now on, we say that two blocks $\mathbf{B}$ and $\mathbf{B}^{\prime}$ in $\mathcal{B}_{n}\left(\Lambda_{N}\right)$ are strongly disjoint if their centers are at a distance of $7 d n$ of each other. Also, 


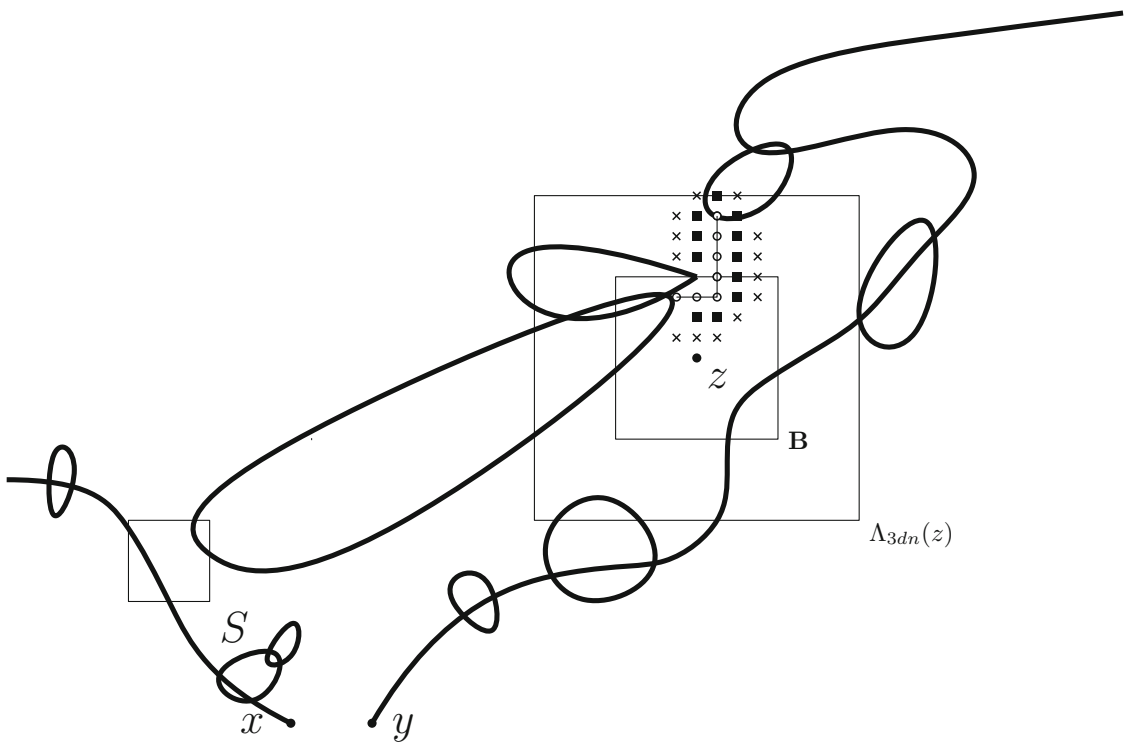

Fig. 2. A schematic version of Lemma 5.2. The thick lines represent the edges in the multigraph underlying $\mathbf{n}_{1}+\mathbf{n}_{2}$ whereas the thin line represents the path $\Pi_{\mathbf{B}}=\left(v_{0}, \ldots, v_{k}\right)$. We also depicted the sets $T_{\mathbf{B}}$ (filled squares) and $S_{\mathbf{B}} \backslash T_{\mathbf{B}} \cup\left\{v_{k}\right\}$ (crosses)

let $N_{r}(S)$ be the set of vertices of $\mathbb{Z}^{d}$ within a graph distance at most $r$ of $S$, and $E_{r}(S)$ be the set of edges with both endpoints in $N_{r}(S)$.

Lemma 5.4. Assume that $\partial \mathbf{n}_{1}=\{x, y\}$ and $\partial \mathbf{n}_{2}=\emptyset$. Let $S \subset \Lambda_{N}$ be such that $\mathcal{C}_{\mathbf{n}_{1}+\mathbf{n}_{2}}(x) \cap S \neq \emptyset$ and $\mathcal{C}_{\mathbf{n}_{1}+\mathbf{n}_{2}}(y) \cap S=\emptyset$. Let $Z$ be a family of strongly disjoint blocks of $\mathcal{B}_{n}\left(\Lambda_{N}\right)$ with the property that every $\mathbf{B} \in Z$ intersects $\mathcal{C}_{\mathbf{n}_{1}+\mathbf{n}_{2}}(S)$ and contains a vertex of $\mathcal{C}_{\mathbf{n}_{1}}(y)$ having at least two neighbors in $\mathcal{C}_{\mathbf{n}_{1}}(y)$.

Then, there exists a new pair of currents $\left(\mathbf{n}_{1}^{\prime}, \mathbf{n}_{2}^{\prime}\right.$ ) (which is a function of $\mathbf{n}_{1}, \mathbf{n}_{2}$ and $Z)$ with the following properties. If the paths $\Pi_{\mathbf{B}}=\Pi_{\mathbf{B}}\left(\mathbf{n}_{1}, \mathbf{n}_{2}\right)=\left(v_{0}^{\mathbf{B}}, v_{1}^{\mathbf{B}}, \ldots, v_{k_{\mathbf{B}}}^{\mathbf{B}}\right)$ are given by Lemma 5.2 above, we have that

(a) $\partial \mathbf{n}_{1}^{\prime}=\partial \mathbf{n}_{1}$ and $\partial \mathbf{n}_{2}^{\prime}=\partial \mathbf{n}_{2}$.

(b) $\left(\mathbf{n}_{1}, \mathbf{n}_{2}\right)(e) \neq\left(\mathbf{n}_{1}^{\prime}, \mathbf{n}_{2}^{\prime}\right)(e)$ implies that $e \in E_{2}\left(\Pi_{\mathbf{B}}\right)$ for some $\mathbf{B} \in Z$.

(c) $\left(\mathbf{n}_{1}, \mathbf{n}_{2}\right)(e) \neq\left(\mathbf{n}_{1}^{\prime}, \mathbf{n}_{2}^{\prime}\right)(e)$ implies that $\mathbf{n}_{j}^{\prime}(e) \leq \mathbf{n}_{j}(e)$ or $\mathbf{n}_{j}^{\prime}(e) \leq 2$ for $j=1,2$.

(d) The set of vertices $v \in \mathcal{C}_{\mathbf{n}_{1}^{\prime}}(y)$ that are connected by $\mathbf{n}_{1}^{\prime}+\mathbf{n}_{2}^{\prime}$ to $S$ in $\left(\Lambda_{N} \backslash \mathcal{C}_{\mathbf{n}_{1}^{\prime}(y)}\right) \cup\{v\}$

is exactly equal to the set of endpoints $v_{k_{\mathbf{B}}}^{\mathbf{B}}$ of the paths $\Pi_{\mathbf{B}}$ with $\mathbf{B} \in Z$.

We already explained why Properties (b) and (d) are important for the reconstruction step. Property (c) is motivated by the observation that

$$
\sum_{\mathbf{n}: \mathbf{n} \geq \mathbf{n}^{\prime}, \mathbf{n}_{\mid \mathrm{E}}=\mathbf{n}_{\mid \mathrm{E}}^{\prime}} \frac{w(\mathbf{n})}{w\left(\mathbf{n}^{\prime}\right)} \leq \mathrm{e}^{C|\mathrm{E}|}
$$

for some $C>0$ and any $\mathrm{E} \subset E_{N}$, and thus is crucial for efficient reconstruction. We will prove Lemma 5.4 slightly later, but before that let us show how can it can be used to derive Lemmata 4.3 and 4.4 . 


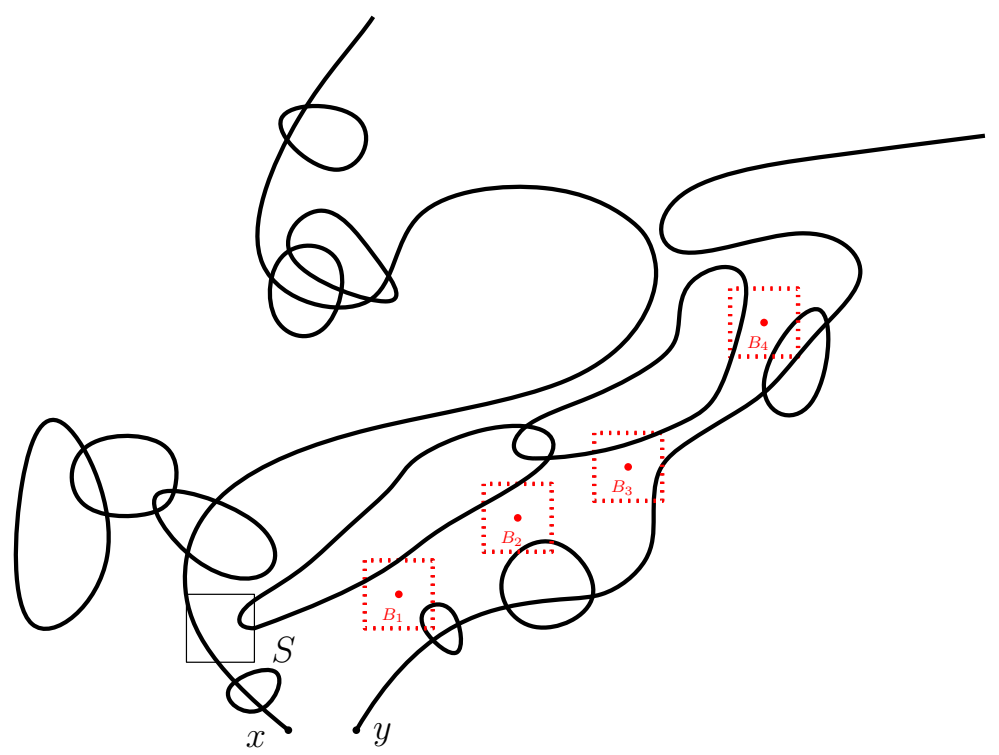

Fig. 3. A simple representation of the set $V\left(\mathbf{n}_{1}, \mathbf{n}_{2}\right)$

Proof of Lemma 4.3. The main idea underlying the proof is the following. We already know from Proposition 4.1 that $\mathbf{B}$ and $\mathbf{B}^{\prime}$ are connected in $\mathbf{n}_{1}+\mathbf{n}_{2}$ for every $\mathbf{B}, \mathbf{B}^{\prime} \in$ $\mathcal{B}_{n}\left(\Lambda_{N}\right)$ with an extremely high probability. As a consequence, we can effectively assume that this happens. Then, it is easy to see that when $\mathcal{C}$ occurs and $x, y$ are not connected in $\Lambda_{N}$ by $\mathbf{n}_{1}+\mathbf{n}_{2}$, one can find a set $S$ and at least $N /(4 n)$ many blocks $\mathbf{B} \in \mathcal{B}_{n}\left(\Lambda_{N}\right)$ satisfying the conditions of Lemma 5.2 (which are the same as the conditions on the elements of $Z$ in Lemma 5.4). Thus, we can pick any subset $Z$ of a certain number of such blocks with the additional condition that they are strongly disjoint, and modify ( $\left.\mathbf{n}_{1}, \mathbf{n}_{2}\right)$ according to the previous lemma. This gives us a relation on the set of pairs of currents and allows us to apply Lemma 5.1 for bounding the probability of the event we are interested in. As we will see later in the proof, the bound we obtain involves the (fixed) size of $Z$ as a parameter and the lemma follows by choosing an appropriate value for this size. The detailed argument is given below.

Construction of $\mathcal{R}$. Let $V\left(\mathbf{n}_{1}, \mathbf{n}_{2}\right)$ be a maximal subset of strongly disjoint blocks $\mathbf{B} \in \mathcal{B}_{n}\left(\Lambda_{N}\right)$ with the property that $\mathcal{C}_{\mathbf{n}_{1}+\mathbf{n}_{2}}(x) \cap \mathbf{B}=\emptyset$ and $\mathbf{B}$ contains a vertex of $\mathcal{C}_{\mathbf{n}_{1}}(y)$ having at least two neighbors in $\mathcal{C}_{\mathbf{n}_{1}}(y)$. Let $\mathcal{C}_{m}$ denote the sub-event of $\mathcal{C}$ for which $V\left(\mathbf{n}_{1}, \mathbf{n}_{2}\right)$ contains exactly $m$ blocks. Also, let $\mathcal{E}$ be the event that every two blocks $\mathbf{B}$ and $\mathbf{B}^{\prime}$ in $\mathcal{B}_{n}\left(\Lambda_{N}\right)$ are connected by $\mathbf{n}_{1}+\mathbf{n}_{2}$ in $\Lambda_{N}$. Finally, define

$$
\mathcal{D}_{m}:=\mathcal{C}_{m} \cap\left\{x \underset{\Lambda_{N}}{\stackrel{\mathbf{n}_{1}+\mathbf{n}_{2}}{\longleftrightarrow}} y\right\} \cap \mathcal{E} .
$$

Let $S=S\left(\mathbf{n}_{1}, \mathbf{n}_{2}\right) \in \mathcal{B}_{n}\left(\Lambda_{N}\right)$ be a block intersecting $\mathcal{C}_{\mathbf{n}_{1}}(x)$ but not $\mathcal{C}_{\mathbf{n}_{1}+\mathbf{n}_{2}}(y)$. We remark that such a set $S$ exists since $\mathcal{D}_{m} \subset \mathcal{C} \subset \mathcal{C}_{y, x}$.

The conditions of Lemma 5.4 are met for $S$ and any subset $Z$ of $V\left(\mathbf{n}_{1}, \mathbf{n}_{2}\right)$. Therefore, we can define a relation as follows. Fix $\delta>0$ to be a small number to be determined later. Then, $\left(\left(\mathbf{n}_{1}, \mathbf{n}_{2}\right),\left(\mathbf{n}_{1}^{\prime}, \mathbf{n}_{2}^{\prime}\right)\right) \in \mathcal{R}$ if and only if $\left(\mathbf{n}_{1}, \mathbf{n}_{2}\right) \in \mathcal{D}_{m}$ and

$$
\left(\mathbf{n}_{1}^{\prime}, \mathbf{n}_{2}^{\prime}\right)=\left(\mathbf{n}_{1}^{\prime}, \mathbf{n}_{2}^{\prime}\right)\left(\mathbf{n}_{1}, \mathbf{n}_{2}, Z\right) \text { for some } Z \subset V\left(\mathbf{n}_{1}, \mathbf{n}_{2}\right) \text { such that }|Z|=\delta m,
$$


where the map $\left(\mathbf{n}_{1}, \mathbf{n}_{2}, Z\right) \mapsto\left(\mathbf{n}_{1}^{\prime}, \mathbf{n}_{2}^{\prime}\right)$ is given by Lemma 5.4.

With this definition of $\mathcal{R}$, we can now check Properties (i) and (ii) of Lemma 5.1 to deduce a bound on the probability of $\mathcal{D}_{m}$.

Property (i). For any $\left(\mathbf{n}_{1}, \mathbf{n}_{2}\right) \in \mathcal{D}_{m}$, the map $Z \mapsto\left\{v_{k_{\mathbf{B}}}^{\mathbf{B}}: \mathbf{B} \in Z\right\}$ is one-to-one since $v_{k_{\mathbf{B}}}^{\mathbf{B}}$ is within a distance $3 d n$ of the center of $\mathbf{B}$, and the blocks in $V\left(\mathbf{n}_{1}, \mathbf{n}_{2}\right)$ are strongly disjoint. Hence,

$$
\left|\mathcal{R}\left(\left(\mathbf{n}_{1}, \mathbf{n}_{2}\right)\right)\right| \geq\left(\begin{array}{c}
\left|V\left(\mathbf{n}_{1}, \mathbf{n}_{2}\right)\right| \\
\delta m
\end{array}\right)=\left(\begin{array}{c}
m \\
\delta m
\end{array}\right) .
$$

Property (ii). Fix $\left(\mathbf{m}_{1}, \mathbf{m}_{2}\right)$. For a block $S$, a set of $\delta m$ strongly disjoint blocks $Z$, a subset of edges $E$ and a collection of paths $\Pi=\left(\Pi_{\mathbf{B}}: \mathbf{B} \in Z\right)$, introduce the set $\mathcal{A}\left(\mathbf{m}_{1}, \mathbf{m}_{2}, S, Z, E, \Pi\right)$ of pairs of currents $\left(\mathbf{n}_{1}, \mathbf{n}_{2}\right) \in \mathcal{D}_{m}$ such that

- $S\left(\mathbf{n}_{1}, \mathbf{n}_{2}\right)=S$,

- $\left(\mathbf{m}_{1}, \mathbf{m}_{2}\right)=\left(\mathbf{n}_{1}^{\prime}, \mathbf{n}_{2}^{\prime}\right)\left(\mathbf{n}_{1}, \mathbf{n}_{2}, Z\right)$,

- $E=\left\{e \in E_{N}:\left(\mathbf{n}_{1}, \mathbf{n}_{2}\right)(e) \neq\left(\mathbf{m}_{1}, \mathbf{m}_{2}\right)(e)\right\}$,

- $\Pi_{\mathbf{B}}\left(\mathbf{n}_{1}, \mathbf{n}_{2}\right)=\Pi_{\mathbf{B}}$ for every $\mathbf{B} \in Z$.

The definition of $\mathcal{R}$ implies directly that

$\sum_{\left(\mathbf{n}_{1}, \mathbf{n}_{2}\right) \in \mathcal{R}^{-1}\left(\mathbf{m}_{1}, \mathbf{m}_{2}\right)} \mathbb{P}_{\Lambda_{N}^{+}, \Lambda_{N}}^{\{x, y\},}\left[\left(\mathbf{n}_{1}, \mathbf{n}_{2}\right)\right]$

$$
=\mathbb{P}_{\Lambda_{N}^{+}, \Lambda_{N}}^{\{x, y\}, \emptyset}\left[\left(\mathbf{m}_{1}, \mathbf{m}_{2}\right)\right] \sum_{(S, Z, E, \Pi)} \sum_{\left(\mathbf{n}_{1}, \mathbf{n}_{2}\right) \in \mathcal{A}\left(\mathbf{m}_{1}, \mathbf{m}_{2}, S, Z, E, \Pi\right)} \frac{w\left(\mathbf{n}_{1}\right) w\left(\mathbf{n}_{2}\right)}{w\left(\mathbf{m}_{1}\right) w\left(\mathbf{m}_{2}\right)} .
$$

We will bound the two summations on the right of (5.2) in two steps. First, Properties (b) and (c) of Lemma 5.4 imply that

$$
\begin{aligned}
& \sum_{\left(\mathbf{n}_{1}, \mathbf{n}_{2}\right) \in \mathcal{A}\left(\mathbf{m}_{1}, \mathbf{m}_{2}, S, Z, E, \Pi\right)} \frac{w\left(\mathbf{n}_{1}\right) w\left(\mathbf{n}_{2}\right)}{w\left(\mathbf{m}_{1}\right) w\left(\mathbf{m}_{2}\right)} \\
& \leq \prod_{\substack{e \in E \\
j=1,2}}\left(\sum_{\ell \geq \mathbf{m}_{j}(e)} \frac{\beta^{\ell} \mathbf{m}_{j}(e) !}{\beta^{\mathbf{m}_{j}(e)} \ell !}+\sum_{0 \leq \ell \leq \mathbf{m}_{j}(e)} \frac{\beta^{\ell} \mathbf{m}_{j}(e) !}{\beta^{\mathbf{m}_{j}(e)} \ell !} \mathbb{I}\left[\mathbf{m}_{j}(e) \leq 2\right]\right) \\
& \leq \exp (C|E|) \leq \exp (C \delta m n) .
\end{aligned}
$$

In the second line, we used that $E$ is included in the union of the $E_{2}\left(\Pi_{\mathbf{B}}\right)$ for $\mathbf{B} \in Z$. Furthermore, $E_{2}\left(\Pi_{\mathbf{B}}\right)$ is the set of vertices at a graph distance at most 2 from $\Pi_{\mathbf{B}}$. According to Lemma 5.2, $\Pi_{\mathbf{B}}$ must be a shortest length path between two vertices within a distance $3 d n$ of the center of $\mathbf{B}$, a fact which implies that its length is smaller than $6 d^{2} n$. Overall, this implies that $|E| \leq 6 d^{2} n|Z| \leq C \delta m n$.

Second, we bound the number of possibilities for $S, Z, E$ and $\Pi$. Obviously, there are fewer than $\left|\mathcal{B}_{n}\left(\Lambda_{N}\right)\right|$ choices for $S$. Property (d) of Lemma 5.4 implies that $\left(\mathbf{m}_{1}, \mathbf{m}_{2}\right)$ and $S$ determine the points $v_{k_{\mathbf{B}}}^{\mathbf{B}}$ for $\mathbf{B} \in Z$. Since these vertices are within a distance $3 d n$ of the centers of the blocks in $Z$, and that there are $\delta m$ blocks in $Z$, this reduces the number of possibilities for $Z$ to $(2 d)^{3 d \delta m}$. Also, each one of the paths $\Pi_{\mathbf{B}}$ is a self-avoiding path of length at most $6 d^{2} n$ ending at $v_{k_{\mathbf{B}}}^{\mathbf{B}}$, and therefore there are at most $(2 d)^{6 d^{2} n \delta m}$ choices 
for the collection of paths $\Pi_{\mathbf{B}}$ with $\mathbf{B} \in Z$. Finally, $E$ being a subset of $\cup_{\mathbf{B} \in Z} E_{2}\left(\Pi_{\mathbf{B}}\right)$, we deduce that the number of possibilities for $E$ is bounded by $2^{25 d^{3} n \delta m}$.

Overall plugging these bounds and (5.3) into (5.2) gives

$$
\sum_{\left(\mathbf{n}_{1}, \mathbf{n}_{2}\right) \in \mathcal{R}^{-1}\left(\mathbf{m}_{1}, \mathbf{m}_{2}\right)} \mathbb{P}_{\Lambda_{N}^{+}, \Lambda_{N}}^{\{x, y\}, \emptyset}\left[\left(\mathbf{n}_{1}, \mathbf{n}_{2}\right)\right] \leq N^{d} \mathrm{e}^{C \delta m n} \mathbb{P}_{\Lambda_{N}^{+}, \Lambda_{N}}^{\{x, y\}, \emptyset}\left[\left(\mathbf{m}_{1}, \mathbf{m}_{2}\right)\right] .
$$

Conclusion of the proof. Plugging the last inequality and (5.1) in Lemma 5.1 gives

$$
\mathbb{P}_{\Lambda_{N}^{+}, \Lambda_{N}}^{\{x, y\}, \emptyset}\left[\mathcal{D}_{m}\right] \leq \frac{C N^{d} \mathrm{e}^{C \delta m n}}{\left(\begin{array}{c}
m \\
\delta m
\end{array}\right)} .
$$

At this stage, recall that $N=e^{n^{\alpha}}$ with $\alpha>1$. An elementary computation shows that choosing $\delta=\mathrm{e}^{-2 C n}$ (which is a valid choice since $\delta m \geq \mathrm{e}^{-2 C n} N /(4 n)=$ $\mathrm{e}^{n^{\alpha}-2 C n} /(4 n)>1$ for every large $\left.n\right)$ gives that

$$
\mathbb{P}_{\Lambda_{N}^{+}, \Lambda_{N}}^{\{x, y\}, \emptyset}\left[\mathcal{D}_{m}\right] \leq \mathrm{e}^{-c \mathrm{e}^{-C n} m n}
$$

for every $m \geq \frac{1}{4(2 d)^{4 d}} N / n$ and $N$ large enough (independent of $m$ ). Since any pair $\left(\mathbf{n}_{1}, \mathbf{n}_{2}\right) \in \mathcal{C}$ must be in one of the $\mathcal{C}_{m}$ for $m \geq \frac{1}{4(2 d)^{4 d}} N / n$, we obtain that

$$
\mathbb{P}_{\Lambda_{N}^{+}, \Lambda_{N}}^{\{x, y, \emptyset}\left[\mathcal{C} \cap\left\{x \underset{\Lambda_{N}}{\stackrel{\mathbf{n}_{1}+\mathbf{n}_{2}}{\leftrightarrows}} y\right\} \cap \mathcal{E}\right] \leq \mathrm{e}^{-c \mathrm{e}^{-C n} N}
$$

by summing over $m$. Now, since $N=\mathrm{e}^{n^{\alpha}}$ for $\alpha<d-1$, it follows from Proposition 4.1 that

$$
\mathbb{P}_{\Lambda_{N}^{+}, \Lambda_{N}}^{\{x, y\}, \emptyset}[\mathcal{E}] \geq 1-C N^{d} \mathrm{e}^{-c n^{d-1}} \geq 1-\mathrm{e}^{-c n^{d-1}}
$$

for every large enough $N$. The result follows readily from this bound and (5.5).

Remark 5.5. We already used the bound $\alpha<d-1$ to deduce Proposition 1.5 from Lemmata 4.3 and 4.4. The end of the previous proof further explains where $1<\alpha<d-1$ is used. Indeed, $\alpha<d-1$ is used to invoke Proposition 4.1. The bound $\alpha>1$ is there to guarantee that the exponential (in $n$ ) finite-energy cost appearing for instance in (5.3) is overcome by the choice of $N$.

Proof of Lemma 4.4. The proof is the same as the previous one, with $S=\{x\}$ instead of $S$ being a block.

We now focus on the proof of Lemma 5.4 using Lemma 5.2.

Proof of Lemma 5.4. We begin with the construction of $\left(\mathbf{n}_{1}^{\prime}, \mathbf{n}_{2}^{\prime}\right)$ and then show that Properties (a)-(d) are satisfied.

Construction of $\left(\mathbf{n}_{1}^{\prime}, \mathbf{n}_{2}^{\prime}\right)$. We proceed in three steps roughly corresponding to the steps that we outlined before Lemma 5.2, albeit in a slightly different order. For clarity of exposition, we will use different notations for the pairs of currents resulting from different steps. As a result, we construct three intermediate currents called $\mathbf{n}_{1}^{0}, \mathbf{n}_{2}^{0}$, and $\mathbf{n}_{2}^{1}$. At the conclusion of each step, we track the sources of the currents constructed in that step as well as the direction in which their value changes from the previous step. This will help us to verify Properties (a) and (c) at the end. 
Also, since the construction will obviously be independent for every $\mathbf{B} \in Z$ (since the blocks are strongly disjoint), we present it only near one prescribed block $\mathbf{B}$, which we remove from the notation for convenience. We therefore write $\Pi=\left(v_{0}, \ldots, v_{k}\right)$. Also, we set $\bar{\Pi}=\left(v_{1}, \ldots, v_{k-1}\right)$ to be the path $\Pi$ minus its endpoints. We will return to the original notation later when we verify the properties of $\left(\mathbf{n}_{1}^{\prime}, \mathbf{n}_{2}^{\prime}\right)$.

Step 1: Closing the edges adjacent to $\Pi$. We want to make both currents 0 on edges adjacent to $\Pi$ except those adjacent to its endpoints. Formally, set

$$
\begin{aligned}
& \mathbf{n}_{1}^{0}(e):= \begin{cases}0 & \text { if } e=\{v, w\} \text { for } v \in \bar{\Pi} \text { and } w \in \Lambda_{N}, \\
\mathbf{n}_{1}(e) & \text { otherwise, }\end{cases} \\
& \mathbf{n}_{2}^{0}(e):= \begin{cases}0 & \text { if } e=\{v, w\} \text { for } v \in \bar{\Pi} \text { and } w \in \Lambda_{N} \backslash \Pi, \\
\mathbf{n}_{2}(e) & \text { otherwise } .\end{cases}
\end{aligned}
$$

In order to track the sources of $\mathbf{n}_{1}^{0}$, let us first notice that vertices in $\bar{\Pi}$ are not sources since they are incident to edges with zero $\mathbf{n}_{1}^{0}$ current only. Since $\bar{\Pi}$ does not intersect $\mathcal{C}_{\mathbf{n}_{1}}(y)$ or $\mathcal{C}_{\mathbf{n}_{1}+\mathbf{n}_{2}}(S)$ (by the definition of $\Pi$ in Lemma 5.2), we deduce that the sources of $\mathbf{n}_{1}^{0}$ not equal to $x$ or $y$ and within a distance of $4 d n$ of the center of $\mathbf{B}$ must be at a distance exactly equal to 1 of $\bar{\Pi}$ and that at least one of the edges incident to them must have changed value between $\mathbf{n}_{1}$ and $\mathbf{n}_{1}^{0}$. Since edges between $\bar{\Pi}$ and $\mathcal{C}_{\mathbf{n}_{1}+\mathbf{n}_{2}}(S) \cup \mathcal{C}_{\mathbf{n}_{1}}(y)$ already had a zero current in $\mathbf{n}_{1}$, we deduce that the new sources cannot be in $\mathcal{C}_{\mathbf{n}_{1}+\mathbf{n}_{2}}(S)$ and must therefore be in the set $T_{\mathbf{B}}=T_{\mathbf{B}}\left(\mathbf{n}_{1}, \mathbf{n}_{2}\right)$ defined in Lemma 5.2. Also, notice that there is an even number of such sources. Similarly, we get that the set of sources of $\mathbf{n}_{2}^{0}$ is a subset of $\bar{\Pi} \cup T_{\mathbf{B}}$.

Finally, notice that

$$
\mathbf{n}_{1}^{0}(e) \leq \mathbf{n}_{1}(e) \text { and } \mathbf{n}_{2}^{0}(e) \leq \mathbf{n}_{2}(e) \text { for every } e \in E_{N} .
$$

Step 2. Opening the edges along $\Pi$. The second step consists in defining $\mathbf{n}_{1}^{1}=\mathbf{n}_{1}^{0}$ and

$$
\mathbf{n}_{2}^{1}(e):= \begin{cases}2 & \text { if } e=\{v, w\} \text { with } v, w \in \Pi \text { or if } v=v_{k} \text { and } w \in \Lambda_{N} . \\ \mathbf{n}_{2}^{0}(e) & \text { otherwise. }\end{cases}
$$

By definition,

$$
\mathbf{n}_{2}^{1}(e) \neq \mathbf{n}_{2}^{0}(e) \text { implies that } \mathbf{n}_{2}^{1}(e)=2 .
$$

Since $\bar{\Pi}$ does not intersect $\mathcal{C}_{\mathbf{n}_{1}+\mathbf{n}_{2}}(S)$, we have $\mathbf{n}_{2}\left(\left\{v_{k-1}, v_{k}\right\}\right)=\mathbf{n}_{2}^{0}\left(\left\{v_{k-1}, v_{k}\right\}\right)=0$ and therefore the definition of $\mathbf{n}_{2}^{1}$ also implies that a source of $\mathbf{n}_{2}^{1}$ is a source of $\mathbf{n}_{2}^{0}$ which is not on $\Pi$. Hence, it is included in $T_{\mathbf{B}}$ again and also, there are an even number of sources within a distance of $4 d n$ of $\mathbf{B}$.

Step 3. Killing the sources of $\left(\mathbf{n}_{1}^{1}, \mathbf{n}_{2}^{1}\right)$. We now remove the additional sources of $\mathbf{n}_{1}^{1}$ and $\mathbf{n}_{2}^{1}$, all of which lie in $T_{\mathbf{B}}$. We start with the sources of $\mathbf{n}_{1}^{1}$. By the fifth item of Lemma 5.2, $T_{\mathbf{B}}$ is a connected subset of $S_{\mathbf{B}}$. We can therefore choose (in some arbitrary but fixed manner) paths $\Gamma_{1}^{1}, \ldots, \Gamma_{\ell}^{1}$ in $S_{\mathbf{B}}$ pairing the sources of $\mathbf{n}_{1}^{1}$. Let $\mathbf{m}$ be the current, equal at each edge to 0 (resp. 1) if there is an even (resp. odd) number of paths going through this edge. Finally, set

$$
\mathbf{n}_{1}^{\prime}(e):= \begin{cases}\mathbf{n}_{1}^{1}(e)-\mathbf{m}(e) & \text { if } \mathbf{n}_{1}^{1}(e) \geq 2 \\ \mathbf{n}_{1}^{1}(e)+\mathbf{m}(e) & \text { otherwise }\end{cases}
$$


We obtain immediately that $\partial \mathbf{n}_{1}^{\prime}=\{x, y\}$. We proceed in the same way for $\mathbf{n}_{2}^{1}$ in order to obtain $\mathbf{n}_{2}^{\prime}$ with $\partial \mathbf{n}_{2}^{\prime}=\emptyset$. Again, notice that

$$
\mathbf{n}_{j}^{\prime}(e) \leq \mathbf{n}_{j}^{1}(e) \text { or } \mathbf{n}_{j}^{\prime}(e) \leq 2 \text { for every } e \in E_{N} \text { and } j=1,2 .
$$

Verification of the properties of $\left(\mathbf{n}_{1}^{\prime}, \mathbf{n}_{2}^{\prime}\right)$. In this part, we assume that we made the construction above for every $\mathbf{B} \in Z$.

Property (a) This follows readily from the two sentences preceding (5.11).

Property (b) At each stage of the construction, edges for which the value of currents is modified are always within a graph distance 2 from one of the paths $\Pi_{\mathbf{B}}$, which means that Property (b) is satisfied.

Property (c) This follows from the displays (5.9), (5.10) and (5.11).

Property (d) Let us first verify one direction of Property (d), namely that for any $\mathbf{B} \in Z$, $v_{k_{\mathbf{B}}}^{\mathbf{B}} \in \mathcal{C}_{\mathbf{n}_{1}^{\prime}}(y)$ and it is connected in $\left(\Lambda_{N} \backslash \mathcal{C}_{\mathbf{n}_{1}^{\prime}(y)}\right) \cup\left\{v_{k_{\mathbf{B}}}^{\mathbf{B}}\right\}$ to $S$ by $\mathbf{n}_{1}^{\prime}+\mathbf{n}_{2}^{\prime}$. We divide the proof into two steps.

Proof that $v_{k_{\mathbf{B}}}^{\mathbf{B}} \in \mathcal{C}_{\mathbf{n}_{1}^{\prime}}(y)$. It suffices to show that $\mathcal{C}_{\mathbf{n}_{1}}(y) \subset \mathcal{C}_{\mathbf{n}_{1}^{\prime}}(y)$. It is clear from our construction in Step 3 that $\mathcal{C}_{\mathbf{n}_{1}^{0}}(y)=\mathcal{C}_{\mathbf{n}_{1}^{1}}(y) \subset \mathcal{C}_{\mathbf{n}_{1}^{\prime}}(y)$. Also, the definition of $\mathbf{n}_{1}^{0}$ implies that $\mathbf{n}_{1}^{0}(e)<\mathbf{n}_{1}(e)$ only if $e=\{v, w\}$ for $v \in \bar{\Pi}_{\mathbf{B}}$ where $\mathbf{B} \in Z$ and $w \in \Lambda_{N}$. Since $\bar{\Pi}_{\mathbf{B}}$ does not intersect $\mathcal{C}_{\mathbf{n}_{1}}(y)$ by Lemma 5.2, we therefore get $\mathcal{C}_{\mathbf{n}_{1}^{0}}(y)=\mathcal{C}_{\mathbf{n}_{1}}(y)$ which completes the proof.

Proof that $v_{k_{\mathbf{B}}}^{\mathbf{B}}$ is connected in $\left(\Lambda_{N} \backslash \mathcal{C}_{\mathbf{n}_{1}^{\prime}(y)}\right) \cup\left\{v_{k_{\mathbf{B}}}^{\mathbf{B}}\right\}$ to $S$ by $\mathbf{n}_{1}^{\prime}+\mathbf{n}_{2}^{\prime}$. Notice that the path $\Pi_{\mathbf{B}}$ is open in $\mathbf{n}_{2}^{\prime}$ and connects $v_{k_{\mathbf{B}}}^{\mathbf{B}}$ to $v_{0}^{\mathbf{B}}$. Since $v_{0}^{\mathbf{B}} \in \mathcal{C}_{\mathbf{n}_{1}+\mathbf{n}_{2}}(S)$ by definition, it therefore suffices to show that $\mathcal{C}_{\mathbf{n}_{1}+\mathbf{n}_{2}}(S) \subset \mathcal{C}_{\mathbf{n}_{1}^{\prime}+\mathbf{n}_{2}^{\prime}}(S)$ and that $\bar{\Pi}_{\mathbf{B}}$ and $\mathcal{C}_{\mathbf{n}_{1}+\mathbf{n}_{2}}(S)$ do not intersect $\mathcal{C}_{\mathbf{n}_{1}^{\prime}}(y)$.

To this end, let us first recall from the definitions of $\left(\mathbf{n}_{1}^{1}, \mathbf{n}_{2}^{1}\right)$ and $\left(\mathbf{n}_{1}^{\prime}, \mathbf{n}_{2}^{\prime}\right)$ that $\left(\mathbf{n}_{1}^{\prime}+\right.$ $\left.\mathbf{n}_{2}^{\prime}\right)(e)>0$ whenever $\left(\mathbf{n}_{1}^{0}+\mathbf{n}_{2}^{0}\right)(e)>0$, and consequently $\mathcal{C}_{\mathbf{n}_{1}^{0}+\mathbf{n}_{2}^{0}}(S) \subset \mathcal{C}_{\mathbf{n}_{1}^{\prime}+\mathbf{n}_{2}^{\prime}}(S)$. Also, from the construction in Step 1 we have $\left(\mathbf{n}_{1}^{0}+\mathbf{n}_{2}^{0}\right)(e)<\left(\mathbf{n}_{1}+\mathbf{n}_{2}\right)(e)$ only if $e$ has an endpoint in $\bar{\Pi}_{\mathbf{B}}$ for some $\mathbf{B} \in Z$. Since $\bar{\Pi}_{\mathbf{B}}$ does not intersect $\mathcal{C}_{\mathbf{n}_{1}+\mathbf{n}_{2}}(S)$ by Lemma 5.2, it therefore follows that $\mathcal{C}_{\mathbf{n}_{1}^{0}+\mathbf{n}_{2}^{0}}(S)=\mathcal{C}_{\mathbf{n}_{1}+\mathbf{n}_{2}}(S)$ and hence $\mathcal{C}_{\mathbf{n}_{1}+\mathbf{n}_{2}}(S) \subset \mathcal{C}_{\mathbf{n}_{1}^{\prime}+\mathbf{n}_{2}^{\prime}}(S)$.

Next let us "bound" the set $\mathcal{C}_{\mathbf{n}_{1}^{\prime}}(y)$ from above. Notice that

$$
\mathcal{C}_{\mathbf{n}_{1}^{\prime}}(y) \subset \mathcal{C}_{\mathbf{n}_{1}^{0}}(y) \cup \bigcup_{\mathbf{B} \in Z} \mathcal{C}_{\mathbf{n}_{1}^{0}}\left(S_{\mathbf{B}}\right)=\mathcal{C}_{\mathbf{n}_{1}}(y) \cup \bigcup_{\mathbf{B} \in Z} \mathcal{C}_{\mathbf{n}_{1}^{0}}\left(S_{\mathbf{B}}\right),
$$

where in the second step we used $\mathcal{C}_{\mathbf{n}_{1}}(y)=\mathcal{C}_{\mathbf{n}_{1}^{0}}(y)$ as proved in the previous part. Since, by Lemma 5.2, $\bar{\Pi}_{\mathbf{B}}$ is disjoint from $\mathcal{C}_{\mathbf{n}_{1}}(y)$ and $S_{\mathbf{B}}$, we deduce from the previous displayed equation that $\mathcal{C}_{\mathbf{n}_{1}^{\prime}}(y) \cap \bar{\Pi}_{\mathbf{B}}=\emptyset$ for any $\mathbf{B} \in Z$ if we show $\mathcal{C}_{\mathbf{n}_{1}^{0}}\left(\bar{\Pi}_{\mathbf{B}}\right)=\bar{\Pi}_{\mathbf{B}}$ for any such $\mathbf{B}$. But this follows immediately from the fact that $\mathbf{n}_{1}^{0}(e)=0$ for any edge $e$ whose one endpoint lies in $\bar{\Pi}_{\mathbf{B}}$ for some $\mathbf{B} \in Z$.

Similarly, from $\mathcal{C}_{\mathbf{n}_{1}}(y) \cap \mathcal{C}_{\mathbf{n}_{1}+\mathbf{n}_{2}}(S)=\emptyset$ and $S_{\mathbf{B}} \cap \mathcal{C}_{\mathbf{n}_{1}+\mathbf{n}_{2}}(S)=S_{\mathbf{B}} \cap \mathcal{C}_{\mathbf{n}_{1}^{0}+\mathbf{n}_{2}^{0}}(S)=$ $\emptyset$ (both are consequences of Lemma 5.2), we deduce that $\mathcal{C}_{\mathbf{n}_{1}^{\prime}}(y) \cap \mathcal{C}_{\mathbf{n}_{1}+\mathbf{n}_{2}}(S)=\emptyset$, concluding the proof of this part.

It remains to verify the other direction of Property (d), namely that any vertex $v \in$ $\mathcal{C}_{\mathbf{n}_{1}^{\prime}}(y)$ that is connected to $S$ by $\mathbf{n}_{1}^{\prime}+\mathbf{n}_{2}^{\prime}$ in $\left(\Lambda_{N} \backslash \mathcal{C}_{\mathbf{n}_{1}^{\prime}(y)}\right) \cup\{v\}$ must be the endpoint $v_{k_{\mathbf{B}}}^{\mathbf{B}}$ of a path $\Pi_{\mathbf{B}}$ for some $\mathbf{B} \in Z$. It suffices to show that any self-avoiding path $\Pi$ in $\mathbf{n}_{1}^{\prime}+\mathbf{n}_{2}^{\prime}$ between $S$ and $\mathcal{C}_{\mathbf{n}_{1}^{\prime}}(y)$ contains $v_{k_{\mathbf{B}}}^{\mathbf{B}}$ for some $\mathbf{B} \in Z$. 
Since the blocks are strongly disjoint, we deduce from the definition of $\left(\mathbf{n}_{1}^{\prime}, \mathbf{n}_{2}^{\prime}\right)$ that

$$
\left(\mathbf{n}_{1}^{\prime}+\mathbf{n}_{2}^{\prime}\right)(e)=\left(\mathbf{n}_{1}^{1}+\mathbf{n}_{2}^{1}\right)(e)=0
$$

for every edge with one endpoint in some $\bar{\Pi}_{\mathbf{B}}$. Together with the fact that $\bar{\Pi}_{\mathbf{B}}$ is disjoint from $\mathcal{C}_{\mathbf{n}_{1}^{\prime}}(y)$ and $S$ (already noted in the previous parts), this implies that if $\Pi$ contains an edge of $\Pi_{\mathbf{B}}$, then it must contain the vertex $v_{k_{\mathbf{B}}}^{\mathbf{B}}$. On the other hand, if $\Pi$ does not contain any edge of $\Pi_{\mathbf{B}}$ for any $\mathbf{B} \in Z$, then it must contain an edge with one endpoint in $\mathcal{C}_{\mathbf{n}_{1}+\mathbf{n}_{2}}(S)$ and another which is not in $\mathcal{C}_{\mathbf{n}_{1}+\mathbf{n}_{2}}(S)$ or in any of the $\bar{\Pi}_{\mathbf{B}}$. This is because $\mathcal{C}_{\mathbf{n}_{1}^{\prime}}(y) \cap \mathcal{C}_{\mathbf{n}_{1}+\mathbf{n}_{2}}(S)=\emptyset$ as we noted in the previous part. Obviously such an edge cannot exist in $\mathbf{n}_{1}+\mathbf{n}_{2}$. Now, observing that $\left(\mathbf{n}_{1}+\mathbf{n}_{2}\right)(e)<\left(\mathbf{n}_{1}^{\prime}+\mathbf{n}_{2}^{\prime}\right)(e)$ only if $e$ is an edge in $\Pi_{\mathbf{B}}$ or $S_{\mathbf{B}}$ for some $\mathbf{B} \in Z$ and that $S_{\mathbf{B}} \cap \mathcal{C}_{\mathbf{n}_{1}+\mathbf{n}_{2}}(S)=\emptyset$, we conclude that such an edge cannot exist in $\mathbf{n}_{1}^{\prime}+\mathbf{n}_{2}^{\prime}$ as well, thus finishing the proof.

Finally, we are left with the proof of Lemma 5.2. It is clear that the only non-trivial part of the lemma is the fifth item. However, the following crucial observation makes it much simpler. Suppose that we choose $\Pi_{\mathbf{B}}:=\left(v_{0}, \ldots, v_{k}\right)$ as a shortest path between $\mathcal{C}_{\mathbf{n}_{1}+\mathbf{n}_{2}}(S)$ and $\mathcal{C}_{\mathbf{n}_{1}}(y)$ restricted to some region. Also suppose that $N_{2}\left(\Pi_{\mathbf{B}}\right)$ lies in that region. Then the distance between $v_{t}$ and $\mathcal{C}_{\mathbf{n}_{1}+\mathbf{n}_{2}}(S)$ is at least 3 for any $t \geq 3$ (since otherwise there would be a shorter path between $\mathcal{C}_{\mathbf{n}_{1}+\mathbf{n}_{2}}(S)$ and $\mathcal{C}_{\mathbf{n}_{1}}(y)$ restricted to the region) and hence $N_{2}\left(v_{t}\right) \backslash\left(\Pi_{\mathbf{B}} \cup \mathcal{C}_{\mathbf{n}_{1}+\mathbf{n}_{2}}(S)\right)=N_{2}\left(v_{t}\right) \backslash \Pi_{\mathbf{B}}$ for any such $t$. Now, in dimension $d \geq 3$, it is not difficult to prove that $N_{1}\left(v_{t}\right) \backslash \Pi_{\mathbf{B}}$ is a connected subset of $N_{2}\left(v_{t}\right) \backslash \Pi_{\mathbf{B}}$ if $\Pi_{\mathbf{B}}$ is a shortest path between its endpoints. Hence, any two vertices in $N_{1}\left(v_{t}\right) \backslash \Pi_{\mathbf{B}}$ can be connected using a path in $N_{2}\left(v_{t}\right) \backslash \Pi_{\mathbf{B}}$ that does not intersect $\mathcal{C}_{\mathbf{n}_{1}+\mathbf{n}_{2}}(S)$. Therefore the only "problematic" vertices in $T_{\mathbf{B}}$ (see the statement of Lemma 5.2) are those in $N_{1}\left(v_{1}\right)$ and $N_{1}\left(v_{2}\right)$. We deal with them in the proof by carefully considering all possible alignments for the first three edges of $\Pi_{\mathbf{B}}$.

Proof of Lemma 5.2. For the purpose of this proof, we use $\mathbf{e}_{j}$ to denote the vertex in $\mathbb{Z}^{d}$ whose $j$-th coordinate is 1 and all the other coordinates are 0 . Let $u$ be a vertex in $\mathcal{C}_{\mathbf{n}_{1}}(y) \cap \mathbf{B}$ with at least two neighbors in $\mathcal{C}_{\mathbf{n}_{1}}(y)$. Since $\mathbf{B}$ intersects $\mathcal{C}_{\mathbf{n}_{1}+\mathbf{n}_{2}}(S)$, there is a vertex $v \in \mathcal{C}_{\mathbf{n}_{1}+\mathbf{n}_{2}}(S)$ which is within a distance of $3 d n$ from the center of $\mathbf{B}$ realizing the graph distance, denoted by $d_{1}$ below, between $u$ and $\mathcal{C}_{\mathbf{n}_{1}+\mathbf{n}_{2}}(S)$ restricted to the box of radius $4 d n$ with the same center as $\mathbf{B}$.

Now consider a shortest (for the length) path $\Pi^{\prime}:=\left(v=v_{0}^{\prime}, v_{1}^{\prime}, \ldots, v_{d_{1}}^{\prime}=u\right)$ between $v$ and $u$ as our first choice for $\Pi_{\mathbf{B}}$. For any two vertices $p, q$ adjacent to $v_{t}$ which do not lie in $\Pi^{\prime}$ or $\mathcal{C}_{\mathbf{n}_{1}+\mathbf{n}_{2}}(S)$ (where $t \geq 1$ ), we would like to connect them by a path in $\left\{v_{d_{1}}^{\prime}\right\} \cup N_{2}\left(\Pi^{\prime}\right) \backslash \Pi^{\prime}$ which avoids $\mathcal{C}_{\mathbf{n}_{1}+\mathbf{n}_{2}}(S)$. In what follows we do this based on the value of $t$.

When $t \geq 3$, the observation we made before the proof implies that the graph distance between any vertex in $N_{2}\left(v_{t}^{\prime}\right)$ and $u$ is strictly less than $d_{1}$ and hence $N_{2}\left(v_{t}^{\prime}\right)$ does not intersect $\mathcal{C}_{\mathbf{n}_{1}+\mathbf{n}_{2}}(S)$. Thus we only need to show that, for $t \geq 3$, the set $N_{1}\left(v_{t}^{\prime}\right) \backslash \Pi^{\prime}$ is connected in $N_{2}\left(v_{t}^{\prime}\right) \backslash \Pi^{\prime}$. To this end we consider two distinct possibilities for a pair of vertices $w, w^{\prime}$ in $N_{1}\left(v_{t}^{\prime}\right) \backslash \Pi^{\prime}$. The first possibility is that $w=v_{t}^{\prime}+\mathbf{e}$ and $w^{\prime}=v_{t}^{\prime}+\mathbf{e}^{\prime}$ for some $\mathbf{e}, \mathbf{e}^{\prime} \in\left\{ \pm e_{j}: j \leq d\right\}$ such that $\mathbf{e} \neq-\mathbf{e}^{\prime}$. Notice that in this case, the vertex $v_{t}^{\prime}+\mathbf{e}+\mathbf{e}^{\prime}$ cannot lie in $\Pi^{\prime}$ since otherwise the segment of $\Pi^{\prime}$ between $v_{t}^{\prime}$ and $v_{t}^{\prime}+\mathbf{e}+\mathbf{e}^{\prime}$ would contain at least 3 edges contradicting the fact that $\Pi^{\prime}$ is a shortest path. Hence the path $\left(w, v_{t}^{\prime}+\mathbf{e}+\mathbf{e}^{\prime}, w^{\prime}\right)$ lies in $N_{2}\left(v_{t}^{\prime}\right) \backslash \Pi^{\prime}$. The second possibility is that $w=v_{t}^{\prime}-\mathbf{e}$ and $w^{\prime}=v_{t}^{\prime}+\mathbf{e}$ for some $\mathbf{e} \in\left\{ \pm \mathbf{e}_{j}: j \leq d\right\}$ which we can assume, without loss of generality, to be $\mathbf{e}_{1}$. Let $\mathbf{e} \in\left\{ \pm \mathbf{e}_{j}: j=2,3\right\}$ and consider the path $\left(w, w+\mathbf{e}, v_{t}^{\prime}+\mathbf{e}, w^{\prime}+\mathbf{e}, w^{\prime}\right)$ 
in $N_{2}\left(v_{t}^{\prime}\right)$. If this path intersects $\Pi^{\prime}$, then our previous argument yields that $v_{t}^{\prime}+\mathbf{e} \in \Pi^{\prime}$. Since $\Pi^{\prime}$ is a self-avoiding path, it cannot contain more than 2 neighbors of $v_{t}^{\prime}$ and thus the path $\left(w, w+\mathbf{e}, v_{t}^{\prime}+\mathbf{e}, w^{\prime}+\mathbf{e}, w^{\prime}\right)$ lies in $N_{2}\left(v_{t}^{\prime}\right) \backslash \Pi^{\prime}$ for some $\mathbf{e} \in\left\{ \pm \mathbf{e}_{j}: j=1,2\right\}$.

Thus it only remains to deal with the vertices adjacent to $v_{1}$ and $v_{2}$ which do not lie in $\Pi^{\prime}$ or $\mathcal{C}_{\mathbf{n}_{1}+\mathbf{n}_{2}}(S)$. Unfortunately it may not be always possible to connect a pair of such vertices by a path in $\left\{v_{d_{1}}^{\prime}\right\} \cup N_{2}\left(\Pi^{\prime}\right) \backslash \Pi^{\prime}$ and in those cases we need to modify $\Pi^{\prime}$. Below we discuss these cases based on all possible values of $d_{\infty}:=\|v-u\|_{\infty}$ and $d_{1}$. One observation that will be particularly useful is that $N_{1}\left(v_{2}^{\prime}\right)$ and $\mathcal{C}_{\mathbf{n}_{1}+\mathbf{n}_{2}}(S)$ are disjoint. Thus we only need to connect any vertex in $N_{1}\left(v_{1}^{\prime}\right) \backslash\left(\Pi^{\prime} \cup \mathcal{C}_{\mathbf{n}_{1}+\mathbf{n}_{2}}(S)\right)$ to a vertex in $N_{1}\left(v_{t}^{\prime}\right) \backslash \Pi^{\prime}$ for some $t \geq 2$ and similarly any vertex in $N_{1}\left(v_{2}^{\prime}\right) \backslash \Pi^{\prime}$ to a vertex in $N_{1}\left(v_{t}^{\prime}\right) \backslash \Pi^{\prime}$ for some $t \geq 3$ using a path in $N_{2}\left(\Pi^{\prime}\right) \backslash\left(\Pi^{\prime} \cup \mathcal{C}_{\mathbf{n}_{1}+\mathbf{n}_{2}}(S)\right)$. As a final remark before we go to the details, let us mention that when $d_{1}=1$ or when $d_{\infty}=2$ and $d_{1}=2$, it is not difficult to see that either $\Pi^{\prime}$ or $\left(v_{0}^{\prime}, v_{1}^{\prime}\right)$ satisfies the items of the lemma. Hence, we only focus on the other cases.

Case 1. $d_{\infty} \geq 3$.

In this case we can choose $\Pi^{\prime}$ so that $v_{t}^{\prime}=v_{0}^{\prime}+t$ e for every $t \leq 3$ and some $\mathbf{e} \in\left\{ \pm \mathbf{e}_{j}: j \leq d\right\}$. Without loss of generality, we assume that $\mathbf{e}=\mathbf{e}_{1}$. Notice that for any vertex $p$ in $N_{1}\left(v_{1}^{\prime}\right) \backslash\left(\Pi^{\prime} \cup \mathcal{C}_{\mathbf{n}_{1}+\mathbf{n}_{2}}(S)\right)$, the vertex $p+\mathbf{e}_{1}$ lies in $N_{1}\left(v_{1}^{\prime}+\mathbf{e}_{1}\right) \backslash \Pi^{\prime}=N_{1}\left(v_{2}^{\prime}\right) \backslash \Pi^{\prime}$ and hence the edge $\left\{p, p+\mathbf{e}_{1}\right\}$ lies in $N_{1}\left(\Pi^{\prime}\right) \backslash\left(\Pi^{\prime} \cup \mathcal{C}_{\mathbf{n}_{1}+\mathbf{n}_{2}}(S)\right)$ as well. Similarly any vertex in $N_{1}\left(v_{2}^{\prime}\right) \backslash \Pi^{\prime}$ is either a neighbor of $v_{4}^{\prime}$ (when $v_{4}^{\prime}=v_{3}^{\prime} \pm \mathbf{e}_{j}$ for some $j>1$ ) or has a neighbor in $N_{1}\left(v_{2}^{\prime}+\mathbf{e}_{1}\right) \backslash \Pi^{\prime}=N_{1}\left(v_{3}^{\prime}\right) \backslash \Pi^{\prime}$ (when $v_{4}^{\prime}=v_{3}^{\prime}+\mathbf{e}_{1}$ ). These together imply that the items of Lemma 5.2 hold for $\Pi_{\mathbf{B}}=\left(v_{0}^{\prime}, v_{1}^{\prime}, \ldots, v_{t}^{\prime}\right)$, where $t=\min \left\{t^{\prime} \leq d_{1}: v_{t^{\prime}}^{\prime} \in \mathcal{C}_{\mathbf{n}_{1}}(y)\right\}$.

Case 2. $d_{\infty}=2$ and $d_{1} \geq 3$.

This is the most involved case. Here, we can assume without loss of generality that $v_{t}^{\prime}=v_{0}^{\prime}+t \mathbf{e}_{1}$ for $t \leq 2$ and $v_{3}^{\prime}=v_{2}^{\prime}+\mathbf{e}_{2}$. Just like in Case 1 , any vertex in $N_{1}\left(v_{1}^{\prime}\right) \backslash\left(\Pi^{\prime} \cup \mathcal{C}_{\mathbf{n}_{1}+\mathbf{n}_{2}}(S)\right)$ has a neighbor in $N_{1}\left(v_{2}^{\prime}\right) \backslash\left(\Pi^{\prime} \cup \mathcal{C}_{\mathbf{n}_{1}+\mathbf{n}_{2}}(S)\right)$. Also notice that for any vertex $p$ in $N_{1}\left(v_{2}^{\prime}\right) \backslash\left(\Pi^{\prime} \cup \mathcal{C}_{\mathbf{n}_{1}+\mathbf{n}_{2}}(S)\right)$ other than $v_{2}^{\prime}-\mathbf{e}_{2}$, the vertex $p+\mathbf{e}_{2}$ lies in $N_{1}\left(v_{3}^{\prime}\right) \backslash \Pi^{\prime}$. Hence we only need a separate treatment for $v_{2}^{\prime}-\mathbf{e}_{2}$, i.e. when it does not lie in $\mathcal{C}_{\mathbf{n}_{1}+\mathbf{n}_{2}}(S)$. To this end we consider several sub-cases based on the neighboring vertices of $v_{2}^{\prime}-\mathbf{e}_{2}$ and the value of $d_{1}$.

Case 2-a. $v_{2}^{\prime}-\mathbf{e}_{2}+\mathbf{e} \notin \mathcal{C}_{\mathbf{n}_{1}+\mathbf{n}_{2}}(S)$ for some $\mathbf{e} \in\left\{ \pm \mathbf{e}_{j}: j \leq d\right\} \backslash\left\{-\mathbf{e}_{1}, \pm \mathbf{e}_{2}\right\}$. Notice that $v_{2}^{\prime}+\mathbf{e} \in N_{1}\left(v_{2}^{\prime}\right) \backslash \Pi^{\prime}$. Since $N_{1}\left(v_{2}^{\prime}\right)$ does not intersect $\mathcal{C}_{\mathbf{n}_{1}+\mathbf{n}_{2}}(S)$, it follows that the path $\left(v_{2}^{\prime}-\mathbf{e}_{2}, v_{2}^{\prime}-\mathbf{e}_{2}+\mathbf{e}, v_{2}^{\prime}+\mathbf{e}\right)$ lies in $N_{2}\left(\Pi^{\prime}\right) \backslash\left(\Pi^{\prime} \cup \mathcal{C}_{\mathbf{n}_{1}+\mathbf{n}_{2}}(S)\right)$. Now $v_{2}^{\prime}+\mathbf{e}$, being a neighbor of $v_{2}^{\prime}$ other than $v_{2}^{\prime}-\mathbf{e}_{2}$, has a neighbor in $N_{1}\left(v_{3}^{\prime}\right) \backslash \Pi^{\prime}$ as we already saw above. Thus, like Case 1, we may choose $\Pi_{\mathbf{B}}=\left(v_{0}^{\prime}, v_{1}^{\prime}, \ldots, v_{t}^{\prime}\right)$ for $t=\min \left\{t^{\prime} \leq d_{1}: v_{t^{\prime}}^{\prime} \in \mathcal{C}_{\mathbf{n}_{1}}(y)\right\}$.

Case 2-b. $v_{2}^{\prime}-\mathbf{e}_{2}+\mathbf{e} \in \mathcal{C}_{\mathbf{n}_{1}+\mathbf{n}_{2}}(S)$ for each $\mathbf{e} \in\left\{ \pm \mathbf{e}_{j}: j \leq d\right\} \backslash\left\{-\mathbf{e}_{1}, \pm \mathbf{e}_{2}\right\}$ and $d_{1}>3$. Let us modify $\Pi^{\prime}$ slightly to obtain a new path $\Pi^{\prime \prime}:=\left(v_{2}^{\prime}-\mathbf{e}_{2}+\mathbf{e}_{1}, v_{2}^{\prime}+\mathbf{e}_{1}, v_{2}^{\prime}+\right.$ $\left.\mathbf{e}_{2}+\mathbf{e}_{1}, v_{3}^{\prime}, \ldots, v_{d_{1}}^{\prime}\right)$ and $\mathcal{C}_{\mathbf{n}_{1}+\mathbf{n}_{2}}(S)$ restricted to the box of side-length $6 d n$ with the same center as $\mathbf{B}$ and $u$. In the same spirit as in the case of $\Pi^{\prime}$, the only problematic vertex in $N_{2}\left(\Pi^{\prime \prime}\right) \backslash\left(\Pi^{\prime \prime} \cup \mathcal{C}_{\mathbf{n}_{1}+\mathbf{n}_{2}}(S)\right)$ is $\left(v_{2}^{\prime}+\mathbf{e}_{2}+\mathbf{e}_{1}\right)+\mathbf{e}_{1}=v_{3}^{\prime}+2 \mathbf{e}_{1}$. Hence we can apply the argument from Case 2 -a to $\Pi^{\prime \prime}$ unless $v_{3}^{\prime}+2 \mathbf{e}_{1}+\mathbf{e} \in \mathcal{C}_{\mathbf{n}_{1}+\mathbf{n}_{2}}(S)$ for each $\mathbf{e} \in\left\{ \pm \mathbf{e}_{j}: j \in[d]\right\} \backslash\left\{ \pm \mathbf{e}_{1},-\mathbf{e}_{2}\right\}$. But in that case, since $d_{1}>3$, there must be a vertex of $\mathcal{C}_{\mathbf{n}_{1}+\mathbf{n}_{2}}(S)$ within a graph distance of 2 of $v_{4}$ and thus at a graph distance strictly smaller than $d_{1}$ of $u$ which contradicts the definition of $d_{1}$.

Case 2-c. $v_{2}^{\prime}-\mathbf{e}_{2}+\mathbf{e} \in \mathcal{C}_{\mathbf{n}_{1}+\mathbf{n}_{2}}(S)$ for each $\mathbf{e} \in\left\{ \pm \mathbf{e}_{j}: j \leq d\right\} \backslash\left\{-\mathbf{e}_{1}, \pm \mathbf{e}_{2}\right\}$ and $d_{1}=3$. In view of our discussion in the previous subcase, the only problematic scenario 
is the following. We have that $v_{t}^{\prime}=v_{0}^{\prime}+t \mathbf{e}_{1}$ for $t \leq 2, u=v_{3}^{\prime}=v_{2}^{\prime}+\mathbf{e}_{2}$ and $u+2 \mathbf{e}_{1}+\mathbf{e} \in \mathcal{C}_{\mathbf{n}_{1}+\mathbf{n}_{2}}(S)$ for each $\mathbf{e} \in\left\{ \pm \mathbf{e}_{j}: j \in[d]\right\} \backslash\left\{ \pm \mathbf{e}_{1},-\mathbf{e}_{2}\right\}$. Now recall that $u$ has at least two neighbors in $\mathcal{C}_{\mathbf{n}_{1}}(y)$ which, we claim, gives us in this case two vertices $w$ and $w^{\prime}$ in $\mathcal{C}_{\mathbf{n}_{1}+\mathbf{n}_{2}}(S)$ and $\mathcal{C}_{\mathbf{n}_{1}}(y)$ respectively such that $w^{\prime}=w+2 \mathbf{e}$ for some $\mathbf{e} \in\left\{ \pm \mathbf{e}_{j}: j \leq d\right\}$ and $w^{\prime}$ is within a distance 1 of $\mathbf{B}$. Then it is straightforward to construct $\Pi_{\mathbf{B}}$ from $w$ and $w^{\prime}$ as we already remarked before starting our case studies. In order to verify our claim, we need to consider three distinct possibilities for the two neighbors of $u$ that lie in $\mathcal{C}_{\mathbf{n}_{1}}(y)$.

(i) $v_{2}^{\prime}=u-\mathbf{e}_{2} \in \mathcal{C}_{\mathbf{n}_{1}}(y)$. In this case we can choose $w=v_{0}^{\prime}$ and $w^{\prime}=v_{2}^{\prime}$.

(iii) $u+\mathbf{e}_{1} \in \mathcal{C}_{\mathbf{n}_{1}}(y)$. Here our choices are $w=v_{2}^{\prime}-\mathbf{e}_{2}+\mathbf{e}_{1}$ and $w^{\prime}=u+\mathbf{e}_{1}$.

(iii) $u+\mathbf{e} \in \mathcal{C}_{\mathbf{n}_{1}}(y)$ for some $\mathbf{e} \in\left\{ \pm \mathbf{e}_{j}: j \leq d\right\} \backslash\left\{ \pm \mathbf{e}_{1},-\mathbf{e}_{2}\right\}$. In this case we choose $w=u+2 \mathbf{e}_{1}+\mathbf{e}$ and $w^{\prime}=u+\mathbf{e}$.

Case 3. $d_{\infty}=1$ and $d_{1} \geq 3$.

In this case, let us assume without loss of generality that $v_{1}^{\prime}=v_{0}^{\prime}+\mathbf{e}_{1}, v_{2}^{\prime}=v_{1}^{\prime}+\mathbf{e}_{2}$ and $v_{3}^{\prime}=v_{2}^{\prime}+\mathbf{e}_{3}$. Notice that any vertex $p$ in $N_{1}\left(v_{1}^{\prime}\right) \backslash\left(\Pi^{\prime} \cup \mathcal{C}_{\mathbf{n}_{1}+\mathbf{n}_{2}}(S)\right)$ other than $v_{1}^{\prime}-\mathbf{e}_{2}$ has a neighbor in $N_{1}\left(v_{2}^{\prime}\right) \backslash \Pi^{\prime}$, namely $p+\mathbf{e}_{2}$. Also noting that the vertices $v_{1}^{\prime}-\mathbf{e}_{2}+\mathbf{e}_{3}$ and $v_{1}^{\prime}+\mathbf{e}_{3}$ lie in $N_{2}\left(v_{3}\right) \backslash \Pi^{\prime}$ and $N_{1}\left(v_{3}\right) \backslash \Pi^{\prime}$ respectively, we deduce that $\left(v_{1}^{\prime}-\mathbf{e}_{2}, v_{1}^{\prime}-\mathbf{e}_{2}+\mathbf{e}_{3}, v_{1}^{\prime}+\mathbf{e}_{3}\right)$ is a path in $N_{2}\left(\Pi^{\prime}\right) \backslash\left(\Pi^{\prime} \cup \mathcal{C}_{\mathbf{n}_{1}+\mathbf{n}_{2}}(S)\right)$ if $v_{1}^{\prime}-\mathbf{e}_{2} \notin \mathcal{C}_{\mathbf{n}_{1}+\mathbf{n}_{2}}(S)$.

As to the vertices in $N_{1}\left(v_{2}^{\prime}\right) \backslash \Pi^{\prime}$, we find by the same reasoning as in the analysis of Case 2-a that the only problematic scenario is $v_{2}^{\prime}-\mathbf{e}_{3} \notin \mathcal{C}_{\mathbf{n}_{1}+\mathbf{n}_{2}}(S)$ and $v_{2}^{\prime}-\mathbf{e}_{3}+\mathbf{e} \in$ $\mathcal{C}_{\mathbf{n}_{1}+\mathbf{n}_{2}}(S)$ for every $\mathbf{e} \in\left\{ \pm \mathbf{e}_{j}: j \leq d\right\} \backslash\left\{-\mathbf{e}_{2}, \pm \mathbf{e}_{3}\right\}$. However, in this scenario we would have $\left\|\left(v_{2}^{\prime}-\mathbf{e}_{3}+\mathbf{e}_{2}\right)-u\right\|_{1}=d_{1}$ whereas $\left\|\left(v_{2}^{\prime}-\mathbf{e}_{3}+\mathbf{e}_{2}\right)-u\right\|_{\infty}=2$, thus reducing the problem to Case 2 with $v_{0}^{\prime}=v_{2}^{\prime}-\mathbf{e}_{3}+\mathbf{e}_{2}$, which belongs to $\mathcal{C}_{\mathbf{n}_{1}+\mathbf{n}_{2}}\left(S^{\prime}\right)$ and is within a distance of at most $3 d n$ of the center of $\mathbf{B}$.

Case 4. $d_{\infty}=1$ and $d_{1}=2$.

Let us assume without loss of generality that $u=v_{0}^{\prime}+\mathbf{e}_{1}+\mathbf{e}_{2}$ and $v_{1}^{\prime}=v_{0}^{\prime}+\mathbf{e}_{1}$. In this case we only need to connect any vertex in $N_{1}\left(v_{1}^{\prime}\right) \backslash\left(\Pi^{\prime} \cup \mathcal{C}_{\mathbf{n}_{1}+\mathbf{n}_{2}}(S)\right)$ to a vertex in $N_{1}(u) \backslash \Pi^{\prime}$. To this end notice that any vertex $p$ in $N_{1}\left(v_{1}^{\prime}\right) \backslash\left(\Pi^{\prime} \cup \mathcal{C}_{\mathbf{n}_{1}+\mathbf{n}_{2}}(S)\right)$ other than $v_{1}^{\prime}-\mathbf{e}_{2}$ has a neighbor $p+\mathbf{e}_{2}$ in $N_{1}(u) \backslash \Pi^{\prime}$. Now if $v_{1}^{\prime}-\mathbf{e}_{2} \notin \mathcal{C}_{\mathbf{n}_{1}+\mathbf{n}_{2}}(S)$ and $u+\mathbf{e}$ is a neighbor of $u \operatorname{in} \mathcal{C}_{\mathbf{n}_{1}}(y)$ which is not $u+\mathbf{e}_{2}$ (recall that there are at least two of them), then it is easy to see that either the path $\left(v_{1}^{\prime}-\mathbf{e}_{2}, v_{1}^{\prime}-\mathbf{e}_{2}+\mathbf{e}, v_{1}^{\prime}+\mathbf{e}\right) \operatorname{lies}$ in $N_{2}\left(\Pi^{\prime}\right) \backslash\left(\Pi^{\prime} \cup \mathcal{C}_{\mathbf{n}_{1}+\mathbf{n}_{2}}(S)\right)$ or there exists a vertex $w$ in $\mathcal{C}_{\mathbf{n}_{1}+\mathbf{n}_{2}}(S)$ satisfying $w=(u+\mathbf{e})+2 \mathbf{e}^{\prime}$ or $w=(u+\mathbf{e})+\mathbf{e}^{\prime}$ for some $\mathbf{e}^{\prime} \in\left\{ \pm \mathbf{e}_{j}: j \leq d\right\}$. In both cases, the choice of $\Pi_{B}$ is clear.

Acknowledgements. This research was supported by the ERC CriBLaM, an IDEX grant from Paris-Saclay, a grant from the Swiss FNS, and the NCCR SwissMAP. We thank Michael Aizenman for close collaboration during the beginning of this project. We thank Senya Shlosman for many useful comments. We are grateful to Sébastien Ott for pointing out the result by Alexander to us which led to the addition of Corollary 1.4. We also thank the anonymous referee for a careful review of the article and many useful comments. The paper was completed when AR was a Ph.D. student at IHES.

Open Access This article is distributed under the terms of the Creative Commons Attribution 4.0 International License (http://creativecommons.org/licenses/by/4.0/), which permits unrestricted use, distribution, and reproduction in any medium, provided you give appropriate credit to the original author(s) and the source, provide a link to the Creative Commons license, and indicate if changes were made.

Publisher's Note Springer Nature remains neutral with regard to jurisdictional claims in published maps and institutional affiliations. 


\section{References}

[ABF87] Aizenman, M., Barsky, D.J., Fernández, R.: The phase transition in a general class of Ising-type models is sharp. J. Stat. Phys. 47(3-4), 343-374 (1987)

[ADCS15] Aizenman, M., Duminil-Copin, H., Sidoravicius, V.: Random currents and continuity of ising model's spontaneous magnetization. Commun. Math. Phys. 334, 719-742 (2015)

[AF86] Aizenman, M., Fernández, R.: On the critical behavior of the magnetization in highdimensional Ising models. J. Stat. Phys. 44(3-4), 393-454 (1986)

[Aiz82] Aizenman, M.: Geometric analysis of $\varphi^{4}$ fields and Ising models. Commun. Math. Phys. 86(1), $1-48$ (1982)

[ADTW18] Aizenman, M., Duminil-Copin, H., Tassion, V., Warzel, S.: Emergent planarity in twodimensional ising models with finite-range interactions (2018). arXiv:1801.04960

[Ale98] Alexander, K.S.: On weak mixing in lattice models. Probab. Theory Relat. Fields 110(4), 441-471 (1998)

[BD12] Beffara, V., Duminil-Copin, H.: The self-dual point of the two-dimensional random-cluster model is critical for $q \geq 1$. Probab. Theory Relat. Fields 153(3-4), 511-542 (2012)

[Bod05] Bodineau, T.: Slab percolation for the Ising model. Probab. Theory Relat. Fields 132(1), 83118 (2005)

[Bod06] Bodineau, T.: Translation invariant Gibbs states for the Ising model. Probab. Theory Relat. Fields 135(2), 153-168 (2006)

[CCN87] Chayes, J.T., Chayes, L., Newman, C.M.: Bernoulli percolation above threshold: an invasion percolation analysis. Ann. Probab. 15, 1272-1287 (1987)

[DP96] Deuschel, J., Pisztora, A.: Surface order large deviations for high-density percolation. Probab. Theory Relat. Fields 104(4), 467-482 (1996)

[Dum17] Duminil-Copin, H.: Lectures on the Ising and Potts models on the hypercubic lattice (2017). arXiv: 1707.00520

[DRT17] Duminil-Copin, H., Raoufi, A., Tassion, V.: Sharp phase transition for the random-cluster and potts models via decision trees (2017). arXiv:1705.03104

[DT16] Duminil-Copin, H., Tassion, V.: A new proof of the sharpness of the phase transition for Bernoulli percolation and the Ising model. Commun. Math. Phys. 343(2), 725-745 (2016)

[DT17] Duminil-Copin, H., Tassion, V.: A note on Schramm's locality conjecture for random-cluster models (2017). arXiv:1707.07626

[DS87] Dobrushin, R.L., Shlosman, S.B.: Completely analytical interactions: constructive description. J. Stat. Phys. 46(5/6), 983-1014 (1987)

[FK72] Fortuin, C.M., Kasteleyn, P.W.: On the random-cluster model. I. Introduction and relation to other models. Physica 57, 536-564 (1972)

[FKG71] Fortuin, C.M., Kasteleyn, P.W., Ginibre, J.: Correlation inequalities on some partially ordered sets. Commun. Math. Phys. 22, 89 (1971)

[GHS70] Griffiths, R.B., Hurst, C.A., Sherman, S.: Concavity of magnetization in Ising ferromagnets in a positive external field. J. Math. Phys. 11, 790 (1970)

[Gr67a] Griffiths, R.B.: Correlations in Ising ferromagnets. I. J. Math. Phys. 8, 478 (1967)

[Gr67b] Griffiths, R.B.: Correlations in Ising ferromagnets. II. external magnetic fields. J. Math. Phys. 8, 484 (1967)

[Gri06] Grimmett, G.: The Random-cluster Model, Grundlehren der Mathematischen Wissenschaften [Fundamental Principles of Mathematical Sciences], vol. 333. Springer, Berlin (2006)

[GM90] Grimmett, G.R., Marstrand, J.M.: The supercritical phase of percolation is well behaved. Proc. R. Soc. Lond. A 430, 439-457 (1990)

[LML72] Lebowitz, J.L., Martin-Löf, A.: On the uniqueness of the equilibrium state for ising spin systems. Commun. Math. Phys. 25, 276-282 (1972)

[LP68] Lebowitz, J.L., Penrose, O.: Analytic and clustering properties of thermodynamic functions and distribution functions for classical lattice and continuum systems. Commun. Math. Phys. 11, 99-124 (1968)

[LS13] Lubetzky, E., Sly, A.: Cutoff for the Ising model on the lattice. Invent. Math. 191, 719-755 (2013)

[LSS97] Liggett, T.M., Schonmann, R.H., Stacey, A.M.: Domination by product measures. Ann. Probab. 25(1), 71-95 (1997)

[LY52] Lee, T.D., Yang, C.N.: Statistical theory of equations of state and phase transitions. II. lattice gas and Ising model. Phys. Rev. 87, 410-419 (1952)

[MOS94] Martinelli, F., Olivieri, E., Schonmann, R.H.: For 2-D lattice spin systems weak mixing implies strong mixing. Commun. Math. Phys. 165, 33-47 (1994)

[MW73] McCoy, B.M., Wu, T.T.: The Two-dimensional Ising Model. Harvard University Press, Cambridge (1973) 
[Pis96] Pisztora, A.: Surface order large deviations for Ising, Potts and percolation models. Probab. Theory Relat. Fields 104(4), 427-466 (1996)

[Rao17] Raoufi, A.: Translation-invariant Gibbs states of Ising model: general setting (2017). arXiv:1710.07608

[Rei00] Reimer, D.: Proof of the Van den Berg-Kesten conjecture. Combin. Probab. Comput. 9, 27-32 (2000)

[Sim80] Simon, B.: Correlation inequalities and the decay of correlations in ferromagnets. Commun. Math. Phys. 77(2), 111-126 (1980)

[vdBK85] van den Berg, J., Kesten, H.: Inequalities with applications to percolation and reliability. J. Appl. Probab. 22, 556-569 (1985)

Communicated by L. Erdos 